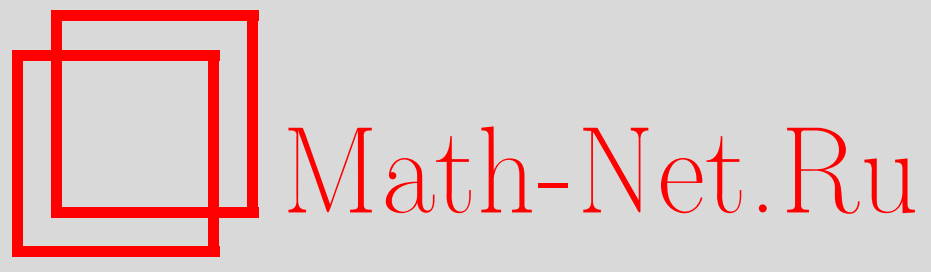

В. В. Провоторов, Собственные функции задачи Штурма-Лиувилля на графе-звезде, Матем. сб., 2008, том 199, номер 10, 105-126

DOI: https://doi.org/10.4213/sm3957

Использование Общероссийского математического портала Math-Net.Ru подразумевает, что вы прочитали и согласны с пользовательским соглашением http://www . mathnet.ru/rus/agreement

Параметры загрузки:

IP: 3.89 .185 .249

26 апреля 2023 г., 14:25:50

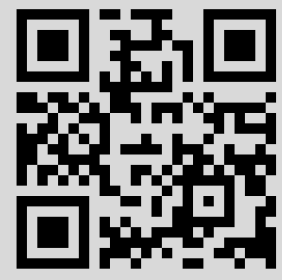




\title{
В. В. Провоторов
}

\section{Собственные функции задачи Штурма-Лиувилля на графе-звезде}

\begin{abstract}
Рассматривается задача Штурма-Лиувилля на графе-звезде. Исследуются структура собственных значений и собственных функций этой задачи и их свойства. Особое внимание уделено вопросам полноты системы собственных функций в пространстве функций с суммируемым квадратом и разложению по этой системе в обобщенный ряд Фурье заданной функции. Эти вопросы являются ключевыми при изучении граничных задач для линейных уравнений в частных производных на графе методом Фурье, возникающих, например, при моделировании колебательных процессов упругой мачты с поддерживающими упругими растяжками.

Библиография: 8 названий.
\end{abstract}

В работе исследуется вопрос обоснования метода разделения переменных для уравнений в частных производных, заданных на графе $\Gamma$, приводящий к важной задаче разложения заданной функции по собственным функциям задачи Штурма-Лиувилля на Г. Основные результаты работы - описание структуры множеств собственных значений и собственных функций, теоремы о полноте и базисности в $L_{2}(\Gamma)$ собственных функций, а также об условиях равномерной сходимости ряда по собственным функциям. Подобные исследования начаты в [1; теоремы 1 и 4], где результат - теорема разложения - сформулирован для истокопредставимых функций. В настоящей работе от разлагаемой функции требуется меньшая гладкость (абсолютная непрерывность на Г). Приведенные исследования лежат в основе изучения прикладных задач, связанных с описанием колебательных процессов, например при моделировании механической системы, состоящей из упругой мачты и поддерживающих ее упругих растяжек [2; соотношения (1)-(6)]. Одна из таких модельных задач приводится ниже. В работе проведено исследование только для случая графа-звезды. Переход к произвольному графу-дереву, которое можно рассматривать как "объединение" конечного числа подграфов, каждый из которых - граф-звезда, осуществляется естественной “склейкой” решений на подграфах и очевиден, хотя сопряжен с некоторыми техническими сложностями.

В дальнейшем будем пользоваться понятиями и обозначениями, использованными в монографии [3; гл. 1, п. 1.3]. Пусть многообразие Г- геометрический граф-звезда с ребрами $\gamma_{k}, k=\overline{1, m}$, и узлом $\xi$; каждое ребро $\gamma_{k}, k=\overline{1, m-1}$, параметризовано отрезком $[0, \pi / 2]$, а ребро $\gamma_{m}$ - отрезком $[\pi / 2, \pi]$, ориентация на ребрах $\gamma_{k}, k=\overline{1, m-1},-$ "к узлу $\xi$ ", на $\gamma_{m}$ - "от узла $\xi$ ". Запись $x \in \gamma_{k}$ означает, что каждой точке $x$ ребра $\gamma_{k}$ придается численное значение $0 \leqslant x \leqslant \pi / 2$ или $\pi / 2 \leqslant x \leqslant \pi, x=\pi / 2 \in \gamma_{k}$ ( $k$ фиксированное, $k=\overline{1, m}$, означает принадлежность точки $x$ узлу $\xi$ ), сужение функции $f(x)$ на ребро $\gamma_{k}$ обозначается

Работа выполнена при поддержке Российского фонда фундаментальных исследований (грант № 07-01-00397).

(C) В. В. Провоторов, 2008 
через $f(x)_{\gamma_{k}}$. Пусть $C(\Gamma)$ - множество непрерывных на $\Gamma$ функций, $C[\Gamma]-$ множество кусочно непрерывных функций (непрерывность на ребрах, пределы в узле $\xi$ по разным ребрам могут быть различными, функции не приписывается никакого значения в узле), $C^{2}[\Gamma]$ - множество функций, все производные которых до второго порядка включительно принадлежат $C[\Gamma]$.

Задача Штурма-Лиувилля на графе $\Gamma$ - это задача поиска функции $y(x) \in$ $C(\Gamma) \cap C^{2}[\Gamma]$, удовлетворяющей уравнениям

$$
-y^{\prime \prime}+q(x) y=\lambda y, \quad x \in \gamma_{k},
$$

на ребрах графа $\gamma_{k}, k=\overline{1, m}$, соотношению в узле $\xi$ (условию согласования [3; гл. 1, п. 1.3])

$$
\sum_{k=1}^{m-1} y^{\prime}\left(\frac{\pi}{2}\right)_{\gamma_{k}}=y^{\prime}\left(\frac{\pi}{2}\right)_{\gamma_{m}}
$$

и краевым условиям

$$
y^{\prime}(0)_{\gamma_{k}}-h_{k} y(0)_{\gamma_{k}}=0, \quad k=\overline{1, m-1}, \quad y^{\prime}(\pi)_{\gamma_{m}}+H y(\pi)_{\gamma_{m}}=0
$$

при некотором $\lambda$, называемом спектральным параметром. Всюду далее будем предполагать, что выполнены следующие условия: $h_{k}, k=\overline{1, m-1}$, и $H$ вещественные; вещественнозначная функция $q(x) \in C(\Gamma)$. Соотношения $(1),(2)$ рассматриваются как реализация единого уравнения на связном множестве $\Gamma$, поэтому их называют уравнением на графе Г [3; гл. 3, п. 3.2].

Предварительно мы изучим свойства собственных значений и собственных функций, установим свойства функции Грина краевой задачи (1)-(3) на основе асимптотических формул для решений уравнения (1), (2).

В дальнейшем нам потребуется следующая известная теорема, доказательство которой можно найти, например, в монографии [4; гл. $1, \S 1.5$, теорема 1.5]. Определим функции $Q_{k}(x), x \in[0, \pi], k=\overline{1, m-1}$, так:

$$
Q_{k}(x) \equiv q(x)_{\gamma_{k}}, \quad x \in\left[0, \frac{\pi}{2}\right], \quad Q_{k}(x) \equiv q(x)_{\gamma_{m}}, \quad x \in\left[\frac{\pi}{2}, \pi\right] .
$$

ТЕОРема 1. Для любого фиксированного $k=\overline{1, m-1}$ и любого а существует единственное решение $z_{k}(x, \lambda) \in C^{1}[0, \pi] \cap C^{2}(0, \pi)$ уравнения

$$
-z_{k}^{\prime \prime}+Q_{k}(x) z_{k}=\lambda z_{k}
$$

такое, что $z_{k}(0, \lambda)=\sin \alpha, z_{k}^{\prime}(0, \lambda)=-\cos \alpha$. Для каждого фиксированного $x \in[0, \pi]$ функция $z_{k}(x, \lambda)$ является целой аналитической функцией от $\lambda$.

Пусть для каждого фиксированного $k, k=\overline{1, m-1}$, функции

$$
\mu_{k}(x, \lambda), \eta_{k}(x, \lambda) \in C^{1}[0, \pi] \cap C^{2}(0, \pi)
$$

- решения уравнения (4), удовлетворяющие начальным условиям $\mu_{k}(\pi / 2, \lambda)=1$, $\mu_{k}^{\prime}(\pi / 2, \lambda)=0, \eta_{k}(\pi / 2, \lambda)=0, \eta_{k}^{\prime}(\pi / 2, \lambda)=1$ соответственно (ясно, что функции $\mu_{k}(x, \lambda), \eta_{k}(x, \lambda)$ при $x \in[\pi / 2, \pi]$ не зависят от индекса $k$, т.е. $\mu_{k}(x, \lambda) \equiv \mu(x, \lambda)$, $\left.\eta_{k}(x, \lambda) \equiv \eta(x, \lambda), x \in[\pi / 2, \pi]\right)$. Тогда функции

$$
\begin{aligned}
\omega_{k}(x, \lambda) & = \begin{cases}\delta_{i k} \eta_{k}(x, \lambda), & x \in \gamma_{i}, i=\overline{1, m-1}, \\
\eta(x, \lambda), & x \in \gamma_{m},\end{cases} \\
\omega_{m}(x, \lambda) & = \begin{cases}\mu_{k}(x, \lambda), & x \in \gamma_{k}, k=\overline{1, m-1}, \\
\mu(x, \lambda), & x \in \gamma_{m}\end{cases}
\end{aligned}
$$


(здесь $\delta_{i k}$ - символ Кронекера), очевидно, являются линейно независимыми решениями уравнения (1), (2).

ЛЕмма 1. Многообразие решений уравнения (1), (2) т-мерно. Тогда

ДокАЗАТЕЛЬСтво. Пусть $y(x, \lambda)$ - произвольное решение уравнения $(1),(2)$.

$$
y(x, \lambda)= \begin{cases}c_{1 k} \mu_{k}(x, \lambda)+c_{2 k} \eta_{k}(x, \lambda), & x \in \gamma_{k}, k=\overline{1, m-1} \\ c_{1 m} \mu(x, \lambda)+c_{2 m} \eta(x, \lambda), & x \in \gamma_{m} .\end{cases}
$$

Непрерывность и гладкость $(2)$ в узле $\xi$ функции $y(x, \lambda)$ связывают постоянные $c_{1 k}, c_{2 k}$ соотношениями $c_{11}=c_{12}=\cdots=c_{1 m}, \sum_{k=1}^{m-1} c_{2 k}=c_{2 m}$. Пусть $c_{2 k}=c_{k}$, $k=\overline{1, m-1}, \quad c_{1 k}=c_{m}, k=\overline{1, m}$. Тогда $c_{2 m}=\sum_{k=1}^{m-1} c_{k}$ и функция $y(x, \lambda)$ в силу представления (6) принимает вид

$$
y(x, \lambda)=\sum_{k=1}^{m} c_{k} \omega_{k}(x, \lambda), \quad x \in \Gamma,
$$

т.е. произвольное решение уравнения (1), (2) является некоторой линейной комбинацией функций $\omega_{k}(x, \lambda), k=\overline{1, m}$.

Систему решений $\left\{\omega_{k}(x, \lambda)\right\}_{1}^{m}$ назовем базовой системой решений уравнения (1), (2).

Пусть для каждого фиксированного $k, k=\overline{1, m-1}$, функции

$$
u_{k}(x, \lambda), v_{k}(x, \lambda) \in C^{1}[0, \pi] \cap C^{2}(0, \pi)
$$

являются решениями уравнения (4) с начальными условиями $u_{k}(0, \lambda)=1$, $u_{k}^{\prime}(0, \lambda)=h_{k}, v_{k}(\pi, \lambda)=1, v_{k}^{\prime}(\pi, \lambda)=-H$. В силу теоремы 1 при каждом фиксированном $\lambda$ функции $u_{k}(x, \lambda), v_{k}(x, \lambda)$ являются целыми аналитическими по $\lambda$, при этом $u_{k}^{\prime}(0, \lambda)-h_{k} u_{k}(0, \lambda)=0, v_{k}^{\prime}(\pi, \lambda)+H v_{k}(\pi, \lambda)=0$ и $v_{k}(x, \lambda)$ при $x \in[\pi / 2, \pi]$ не зависят от индекса $k$, т.е. $v_{k}(x, \lambda) \equiv v(x, \lambda), k=\overline{1, m-1}$, $x \in[\pi / 2, \pi]$. Рассмотрим систему функций

$$
\begin{aligned}
\underset{(k=\overline{1, m-1})}{\varphi_{k}(x, \lambda)} & = \begin{cases}u_{k}\left(\frac{\pi}{2}, \lambda\right) \mu_{i}(x, \lambda), & x \in \gamma_{i}, i=\overline{1, m-1}, i \neq k, \\
u_{k}(x, \lambda), & x \in \gamma_{k} \cup \gamma_{m},\end{cases} \\
\varphi_{m}(x, \lambda) & = \begin{cases}v\left(\frac{\pi}{2}, \lambda\right) \mu_{i}(x, \lambda), & x \in \gamma_{i}, i=\overline{2, m-1}, \\
v(x, \lambda), & x \in \gamma_{1} \cup \gamma_{m} .\end{cases}
\end{aligned}
$$

Очевидно, что функции $\varphi_{k}(x, \lambda), k=\overline{1, m}$, являются решениями уравнения $(1),(2)$ и удовлетворяют краевым условиям $\varphi_{k}^{\prime}(0, \lambda)_{\gamma_{k}}-h_{k} \varphi_{k}(0, \lambda)_{\gamma_{k}}=0$, $k=\overline{1, m-1}, \quad \varphi_{m}^{\prime}(\pi, \lambda)_{\gamma_{m}}+H \varphi_{m}(\pi, \lambda)_{\gamma_{m}}=0$. Покажем их линейную независимость на $Г$.

Предварительно докажем следующее вспомогательное утверждение. Пусть $y_{k}(x, \lambda), k=\overline{1, m},-$ решения уравнения $(1),(2)$ и

$$
y_{k}(x, \lambda)=\sum_{i=1}^{m} a_{k i} \omega_{i}(x, \lambda), \quad k=\overline{1, m},
$$

где $a_{k i}$ - надлежащим образом подобранные константы. 
Лемма 2. Решения $y_{k}(x, \lambda), k=\overline{1, m}$, уравнения (1), (2) линейно независимы на Г тогда и только тогда, когда матрица $\left\|a_{k i}\right\|, k, i=\overline{1, m}$, представлений $y_{k}(x, \lambda)$ в виде (8) через базовую систему решений (5) является невырожденной.

ДокАЗАТЕЛЬСтво. Функции $y_{k}(x, \lambda), k=\overline{1, m}$, линейно независимы, если соотношение $\sum_{k=1}^{m} \alpha_{k} y_{k}(x, \lambda)=0, x \in \Gamma$, имеет место только при $\alpha_{k}=0$, $k=\overline{1, m}$. Учитывая (8), рассмотрим соотношение

$$
\sum_{k=1}^{m} \alpha_{k} \sum_{i=1}^{m} a_{k i} \omega_{i}(x, \lambda)=0, \quad x \in \Gamma .
$$

Так как функции $\omega_{k}(x, \lambda), k=\overline{1, m}$, являются линейно независимыми, то

$$
\sum_{k=1}^{m} \alpha_{k} a_{k i}=0, \quad i=\overline{1, m}
$$

Таким образом, получаем систему линейных однородных уравнений относительно $\alpha_{k}$ с определителем, отличным от нуля. Следовательно, $\alpha_{k}=0, k=$ $\overline{1, m}$. Лемма доказана.

Разложение функций $\varphi_{k}(x, \lambda), k=\overline{1, m}$, по базовой системе (5) имеет следующий вид:

$$
\varphi_{k}(x, \lambda)=\sum_{i=1}^{m} \varphi_{k i} \omega_{i}(x, \lambda), \quad k=\overline{1, m} .
$$

Для каждого фиксированного $k=\overline{1, m}$ в соответствии с (9) получаем $m$ систем линейных алгебраических уравнений относительно постоянных $\varphi_{k i}, k, i=\overline{1, m}$, которые и определяют эти постоянные:

$$
\begin{gathered}
\varphi_{k i}=\delta_{k i} u_{k}^{\prime}\left(\frac{\pi}{2}, \lambda\right), \quad i=\overline{1, m-1}, \quad \varphi_{k m}=u_{k}\left(\frac{\pi}{2}, \lambda\right), \quad k=\overline{1, m-1}, \\
\varphi_{m 1}=v_{1}^{\prime}\left(\frac{\pi}{2}, \lambda\right)=v^{\prime}\left(\frac{\pi}{2}, \lambda\right), \quad \varphi_{m i}=0, \quad i=\overline{2, m-1}, \quad \varphi_{m m}=v\left(\frac{\pi}{2}, \lambda\right) .
\end{gathered}
$$

Определитель матрицы коэффициентов разложений функций $\varphi_{k}(x, \lambda), k=\overline{1, m}$, равен

$$
-\prod_{k=2}^{m-1} u_{k}^{\prime}\left(\frac{\pi}{2}, \lambda\right) \delta_{1}(\lambda)
$$

где

$$
\delta_{1}(\lambda)=u_{1}\left(\frac{\pi}{2}, \lambda\right) v^{\prime}\left(\frac{\pi}{2}, \lambda\right)-u_{1}^{\prime}\left(\frac{\pi}{2}, \lambda\right) v\left(\frac{\pi}{2}, \lambda\right) .
$$

Таким образом, система функций $\left\{\varphi_{k}(x, \lambda)\right\}_{1}^{m}$ является линейно независимой системой решений уравнения (1), (2), если $\lambda$ не является нулем функции

$$
-\prod_{k=2}^{m-1} u_{k}^{\prime}\left(\frac{\pi}{2}, \lambda\right) \delta_{1}(\lambda)
$$

Пусть $(L y)(x) \equiv-y^{\prime \prime}+q(x) y-$ дифференциальное выражение, заданное на многообразии $\Re$, состоящем из функций $y(x) \in C(\Gamma) \cap C^{2}[\Gamma]$, удовлетворяющих условиям согласования (2). 
Обозначим

$$
\left\langle g_{1}(x), g_{2}(x)\right\rangle=g_{1}(x) g_{2}^{\prime}(x)-g_{1}^{\prime}(x) g_{2}(x), \quad x \in \Gamma
$$

(в концевых точках ребер $\gamma_{k}$ применяется одностороннее дифференцирование).

Теорема 2. Для любых функиий $g_{1}(x), g_{2}(x) \in \Re$ имеет место равенство

$$
\begin{gathered}
\int_{\Gamma}\left(L g_{1}\right)(x) g_{2}(x) d x=\left\langle g_{1}(x), g_{2}(x)\right\rangle_{x=\pi}-\sum_{k=1}^{m-1}\left\langle g_{1}(x), g_{2}(x)\right\rangle_{x=0 \in \gamma_{k}} \\
+\int_{\Gamma} g_{1}(x)\left(L g_{2}\right)(x) d x
\end{gathered}
$$

где интеграл на Г определяется как сумма интегралов на $\gamma_{k}, k=\overline{1, m}$.

ДокАЗАтЕльство. Рассмотрим интеграл

$$
\int_{\Gamma}\left(L g_{1}\right)(x) g_{2}(x) d x=\sum_{k=1}^{m} \int_{\gamma_{k}}\left(L g_{1}\right)(x) g_{2}(x) d x .
$$

Вычислим каждый интеграл на $\gamma_{k}$, используя дважды метод интегрирования по частям. Учитывая условие согласования (2), получим

$$
\begin{aligned}
& \sum_{k=1}^{m} \int_{\gamma_{k}}\left(L g_{1}\right)(x) g_{2}(x) d x=\sum_{k=1}^{m} \int_{\gamma_{k}}\left[-g_{1}^{\prime \prime}(x)+q(x) g_{1}(x)\right] g_{2}(x) d x \\
& =\left[g_{1}(x) g_{2}^{\prime}(x)-g_{1}^{\prime}(x) g_{2}(x)\right]_{x=\pi}-\sum_{k=1}^{m-1}\left[g_{1}(x) g_{2}^{\prime}(x)-g_{1}^{\prime}(x) g_{2}(x)\right]_{x=0 \in \gamma_{k}} \\
& \quad+\sum_{k=1}^{m-1}\left[g_{1}(x) g_{2}^{\prime}(x)-g_{1}^{\prime}(x) g_{2}(x)\right]_{x=\pi / 2 \in \gamma_{k}}-\left[g_{1}(x) g_{2}^{\prime}(x)-g_{1}^{\prime}(x) g_{2}(x)\right]_{x=\pi / 2 \in \gamma_{m}} \\
& \quad+\sum_{k=1}^{m} \int_{\gamma_{k}} g_{1}(x)\left(-g_{2}^{\prime \prime}(x)+q(x) g_{2}(x)\right) d x \\
& =\left\langle g_{1}(x), g_{2}(x)\right\rangle_{x=\pi}-\sum_{k=1}^{m-1}\left\langle g_{1}(x), g_{2}(x)\right\rangle_{x=0 \in \gamma_{k}}+\int_{\Gamma} g_{1}(x)\left(L g_{2}\right)(x) d x
\end{aligned}
$$

Формула (10) является аналогом формулы Лагранжа на интервале.

Теорема 3. Собственные значения и собственные функиии краевой задачи (1)-(3) вещественны. Собственные функиии, соответствующие различным собственным значениям, ортогональны в $L_{2}(\Gamma)$.

Доказательство теоремы повторяет классические рассуждения, приводимые на интервале (см., например, [4; гл. 1, §1, теорема 1.1.2]). При этом существенную роль играет формула Лагранжа (10).

Введенные выше функции $u_{k}(x, \lambda), k=\overline{1, m-1}, v(x, \lambda)$ позволяют построить решение $y(x, \lambda)$ краевой задачи $(1)-(3)$ :

$$
y(x, \lambda)= \begin{cases}\alpha_{k} u_{k}(x, \lambda), & x \in \gamma_{k}, k=\overline{1, m-1} \\ \beta v(x, \lambda), & x \in \gamma_{m}\end{cases}
$$


( $\alpha_{k}, \beta$ - некоторые постоянные). Зафиксируем $\lambda$ : пусть $\lambda=\lambda_{0}$. Определение нетривиального решения $y\left(x, \lambda_{0}\right)$ краевой задачи $(1)-(3)$ сводится к определению коэффициентов $\alpha_{k}, k=\overline{1, m-1}$, и $\beta$. В силу непрерывности функции $y_{0}(x)$ в узле $\xi$ получаем

$$
\alpha_{1} u_{k}\left(\frac{\pi}{2}, \lambda_{0}\right)=\alpha_{2} u_{k}\left(\frac{\pi}{2}, \lambda_{0}\right)=\cdots=\alpha_{m-1} u_{k}\left(\frac{\pi}{2}, \lambda_{0}\right),
$$

а условие согласования (2) дает равенство

$$
y_{0}^{\prime}\left(\frac{\pi}{2}\right)_{\gamma_{m}}=\sum_{k=1}^{m-1} \alpha_{k} u_{k}^{\prime}\left(\frac{\pi}{2}, \lambda_{0}\right) .
$$

С другой стороны, $y_{0}(x)_{\gamma_{m}}=\beta v\left(x, \lambda_{0}\right)$, и мы приходим к следующей системе уравнений относительно $\alpha_{k}, k=\overline{1, m-1}$, и $\beta$, одновременно отличных от нуля:

$$
\left\{\begin{array}{l}
\alpha_{k} u_{k}\left(\frac{\pi}{2}, \lambda_{0}\right)=\beta v\left(\frac{\pi}{2}, \lambda_{0}\right), \quad k=\overline{1, m-1} \\
\sum_{k=1}^{m-1} \alpha_{k} u_{k}^{\prime}\left(\frac{\pi}{2}, \lambda_{0}\right)=\beta v^{\prime}\left(\frac{\pi}{2}, \lambda_{0}\right) .
\end{array}\right.
$$

Определитель этой системы $D\left(\lambda_{0}\right)=0$, где

$$
D(\lambda)=v\left(\frac{\pi}{2}, \lambda\right) \sum_{k=1}^{m-1}\left\{u_{k}^{\prime}\left(\frac{\pi}{2}, \lambda\right) \prod_{\substack{i=1 \\ i \neq k)}}^{m-1} u_{i}\left(\frac{\pi}{2}, \lambda\right)\right\}-v^{\prime}\left(\frac{\pi}{2}, \lambda\right) \prod_{i=1}^{m-1} u_{i}\left(\frac{\pi}{2}, \lambda\right) .
$$

Рассмотрим числовое множество $\Lambda(\lambda)=\left\{u_{k}(\pi / 2, \lambda), k=\overline{1, m-1}\right\}$, зависимое от $\lambda$. Пусть $m_{\Lambda}(\lambda)$ - количество его нулевых элементов: $0 \leqslant m_{\Lambda}(\lambda) \leqslant m-1$. Множеству $\Lambda(\lambda)$ соответствует множество коэффициентов $\left\{\alpha_{k}, k=\overline{1, m-1}\right\}$. Заметим, что если при некотором $\lambda_{0}$ выполнено условие $v\left(\pi / 2, \lambda_{0}\right)=0$, то в силу системы $(12) m_{\Lambda}\left(\lambda_{0}\right) \neq 0$, иначе из первых $m-1$ уравнений системы $(12)$ следует, что коэффициенты $\alpha_{k}=0, k=\overline{1, m-1}$, а из последнего уравнения системы получаем, что $\beta=0$. Это означает, что $y\left(x, \lambda_{0}\right)_{\Gamma} \equiv 0$. Аналогично можно показать, что если $v\left(\pi / 2, \lambda_{0}\right) \neq 0$, то $m_{\Lambda}\left(\lambda_{0}\right) \neq 1$.

Пусть $\Omega$ - множество собственных значений краевой задачи (1)-(3); $\Omega^{\mathrm{I}}, \Omega_{\Upsilon}^{\mathrm{II}}$, $\Omega_{\Upsilon}^{\mathrm{III}}-$ множества чисел $\lambda$ следующего вида:

$$
\begin{aligned}
& \Omega^{\mathrm{I}}=\left\{\lambda: v\left(\frac{\pi}{2}, \lambda\right) \neq 0, m_{\Lambda}(\lambda)=0\right\}, \\
& \Omega_{\Upsilon}^{\mathrm{II}}=\left\{\lambda: v\left(\frac{\pi}{2}, \lambda\right) \neq 0, m_{\Lambda}(\lambda)=\Upsilon\right\}, \quad \Upsilon=\overline{2, m-1}, \\
& \Omega_{\Upsilon}^{\mathrm{III}}=\left\{\lambda: v\left(\frac{\pi}{2}, \lambda\right)=0, m_{\Lambda}(\lambda)=\Upsilon\right\}, \quad \Upsilon=\overline{1, m-1} .
\end{aligned}
$$

Теорема 4. Имеет место равенство

$$
\Omega=\Omega^{\mathrm{I}} \cup\left(\bigcup_{\Upsilon=2}^{m-1} \Omega_{\Upsilon}^{\mathrm{II}}\right) \cup\left(\bigcup_{\Upsilon=1}^{m-1} \Omega_{\Upsilon}^{\mathrm{III}}\right)
$$

(множества $\Omega^{\mathrm{I}}, \Omega_{\Upsilon}^{\mathrm{II}}, \Omega_{\Upsilon}^{\mathrm{III}}$ не имеют общих элементов). При этом 
1) если собственное значение $\lambda_{0} \in \Omega^{\mathrm{I}} \cup \Omega_{2}^{\mathrm{II}} \cup \Omega_{1}^{\mathrm{III}}$, то оно простое,

2) если $\lambda_{0} \in \Omega_{\Upsilon}^{\mathrm{II}}, \Upsilon=\overline{3, m-1}$, то его кратность равна $\Upsilon-1$,

3) если $\lambda_{0} \in \Omega_{\Upsilon}^{\mathrm{III}}, \Upsilon=\overline{2, m-1}$, то его кратность равна $\Upsilon$.

ДокАЗАТЕЛЬСтво. Покажем включение

$$
\Omega^{\mathrm{I}} \cup\left(\bigcup_{\Upsilon=2}^{m-1} \Omega_{\Upsilon}^{\mathrm{II}}\right) \cup\left(\bigcup_{\Upsilon=1}^{m-1} \Omega_{\Upsilon}^{\mathrm{III}}\right) \subset \Omega .
$$

1) Пусть $\lambda_{0} \in \Omega^{\mathrm{I}}$. Тогда $u_{k}\left(\pi / 2, \lambda_{0}\right) \neq 0, k=\overline{1, m-1}, v\left(\pi / 2, \lambda_{0}\right) \neq 0$ и постоянные $\alpha_{k}, k=\overline{2, m-1}$, и $\beta$ определяются из системы (12):

$$
\alpha_{k}=\alpha_{1} \frac{u_{1}\left(\pi / 2, \lambda_{0}\right)}{u_{k}\left(\pi / 2, \lambda_{0}\right)}, \quad k=\overline{1, m-1}, \quad \beta=\alpha_{1} \frac{u_{1}\left(\pi / 2, \lambda_{0}\right)}{v\left(\pi / 2, \lambda_{0}\right)} .
$$

Собственная функция $y_{0}(x)$ имеет вид

$$
y_{0}(x)= \begin{cases}\frac{v\left(\pi / 2, \lambda_{0}\right)}{u_{k}\left(\pi / 2, \lambda_{0}\right)} u_{k}\left(x, \lambda_{0}\right), & x \in \gamma_{k}, k=\overline{1, m-1}, \\ v\left(x, \lambda_{0}\right), & x \in \gamma_{m} .\end{cases}
$$

2) Если $\lambda_{0} \in \Omega_{\Upsilon}^{\mathrm{II}}, 2 \leqslant \Upsilon \leqslant m-1$, то $u_{k}\left(\pi / 2, \lambda_{0}\right) \neq 0, k=\overline{1, m-1}, k \neq m_{i}$, $i=\overline{1, \Upsilon}, m_{i}, i=\overline{1, \Upsilon},-$ индексы элементов множества $\Lambda\left(\lambda_{0}\right)$, равных нулю, т.е. $u_{m_{i}}\left(\pi / 2, \lambda_{0}\right)=0, i=\overline{1, \Upsilon}$. Из первых $m-1$ уравнений системы (12) вытекает, что $\alpha_{k}=0, k=\overline{1, m-1}, k \neq m_{i}, i=\overline{1, \Upsilon}, \beta=0, \sum_{i=1}^{\Upsilon} \alpha_{m_{i}} u_{m_{i}}^{\prime}\left(\pi / 2, \lambda_{0}\right)=0$. Представление (11) дает $\Upsilon-1$ собственных функций

$$
y_{0}^{i-1}(x)=\left\{\begin{array}{c}
0, \quad x \in \gamma_{k}, k=\overline{1, m}, \quad k \neq m_{1}, m_{i}, \\
u_{m_{i}}^{\prime}\left(\frac{\pi}{2}, \lambda_{0}\right) u_{m_{1}}\left(x, \lambda_{0}\right), \quad x \in \gamma_{m_{1}}, \\
-u_{m_{1}}^{\prime}\left(\frac{\pi}{2}, \lambda_{0}\right) u_{m_{i}}\left(x, \lambda_{0}\right), \quad x \in \gamma_{m_{i}} .
\end{array}\right.
$$

3) Если $\lambda_{0} \in \Omega_{\Upsilon}^{\mathrm{III}}, 1 \leqslant \Upsilon \leqslant m-1$, то $v\left(\pi / 2, \lambda_{0}\right)=0, u_{k}\left(\pi / 2, \lambda_{0}\right) \neq 0, k=$ $\overline{1, m-1}, k \neq m_{i}, i=\overline{1, \Upsilon}, m_{i}, i=\overline{1, \Upsilon},-$ индексы элементов множества $\Lambda\left(\lambda_{0}\right)$, равных нулю, т.е. $u_{m_{i}}\left(\pi / 2, \lambda_{0}\right)=0, i=\overline{1, \Upsilon}$. Аналогично предыдущему пункту $\alpha_{k}=0, k=\overline{1, m-1}, k \neq m_{i}, i=\overline{1, \Upsilon}, \sum_{i=1}^{\Upsilon} \alpha_{m_{i}} u_{m_{i}}^{\prime}\left(\pi / 2, \lambda_{0}\right)=\beta v\left(\pi / 2, \lambda_{0}\right)$, а представление (11) дает $\Upsilon$ собственных функций

$$
y_{0}^{i}(x)= \begin{cases}0, \quad x \in \gamma_{k}, k=\overline{1, m-1}, & k \neq m_{i}, \\ v^{\prime}\left(\frac{\pi}{2}, \lambda_{0}\right) u_{m_{i}}\left(x, \lambda_{0}\right), & x \in \gamma_{m_{i}}, \\ u_{m_{i}}^{\prime}\left(\frac{\pi}{2}, \lambda_{0}\right) v\left(x, \lambda_{0}\right), & x \in \gamma_{m} .\end{cases}
$$

Пусть теперь $\lambda_{0} \in \Omega$, а $y_{0}(x)$ - соответствующая $\lambda_{0}$ собственная функция. Очевидно, что равенство $y_{0}(0)_{\gamma_{k}}=0, k=\overline{1, m-1}$, невозможно, иначе в силу краевых условий (3) $y_{0}^{\prime}(\pi / 2)_{\gamma_{k}}=0, k=\overline{1, m-1}$, т.е. $y_{0}(x)_{\gamma_{k}}=0, k=\overline{1, m-1}$, и $y_{0}(x)_{\Gamma}=0$. Значит, хотя бы одно из чисел $y_{0}(0)_{\gamma_{k}}, k=\overline{1, m-1}$, отлично от нуля. Учитывая представление решения (11) краевой задачи (1)-(3), будем считать, что для некоторого фиксированного $m_{1}, 1 \leqslant m_{1} \leqslant m-1$, значение 
$y_{0}(0)_{\gamma_{m_{1}}}=y\left(0, \lambda_{0}\right)_{\gamma_{m_{1}}} \neq 0$ и, следовательно, $\alpha_{m_{1}} \neq 0$. Рассмотрим два случая: $u_{m_{1}}\left(\pi / 2, \lambda_{0}\right) \neq 0$ и $u_{m_{1}}\left(\pi / 2, \lambda_{0}\right)=0$.

1. Если $u_{m_{1}}\left(\pi / 2, \lambda_{0}\right) \neq 0$, то $u_{k}\left(\pi / 2, \lambda_{0}\right) \neq 0, k=\overline{1, m-1}, v\left(\pi / 2, \lambda_{0}\right) \neq 0$ (равенство $\prod_{k=1}^{m-1} u_{k}\left(\pi / 2, \lambda_{0}\right) v\left(\pi / 2, \lambda_{0}\right)=0$ невозможно, так как в этом случае в силу системы (11) возникает противоречие: $\left.u_{m_{1}}\left(\pi / 2, \lambda_{0}\right)=0\right)$. Следовательно, $\lambda_{0} \in \Omega^{\mathrm{I}}$.

2. Если $u_{m_{1}}\left(\pi / 2, \lambda_{0}\right)=0$, то возможны такие случаи:

а) $u_{k}\left(\pi / 2, \lambda_{0}\right) \neq 0, k=\overline{1, m-1}, k \neq m_{1}, v\left(\pi / 2, \lambda_{0}\right)=0$ (случай, когда $\prod_{k=1, k \neq m_{1}}^{m-1} u_{k}\left(\pi / 2, \lambda_{0}\right) v\left(\pi / 2, \lambda_{0}\right) \neq 0$, невозможен, так как иначе $\left.y\left(x, \lambda_{0}\right)_{\Gamma} \equiv 0\right)$ и, следовательно, $\lambda_{0} \in \Omega_{1}^{\mathrm{III}}$;

б) $u_{m_{i}}\left(\pi / 2, \lambda_{0}\right)=0,1 \leqslant m_{i} \leqslant m-1, i=\overline{2, \Upsilon}, \Upsilon-$ фиксированное число, $2 \leqslant \Upsilon \leqslant m-1, v\left(\pi / 2, \lambda_{0}\right) \neq 0$, откуда следует, что $\lambda_{0} \in \Omega_{\Upsilon}^{\mathrm{II}}$;

в) $u_{m_{i}}\left(\pi / 2, \lambda_{0}\right)=0,1 \leqslant m_{i} \leqslant m-1, i=\overline{2, \Upsilon}, \Upsilon-$ фиксированное число, $2 \leqslant \Upsilon \leqslant m-1, v\left(\pi / 2, \lambda_{0}\right)=0$, откуда следует, что $\lambda_{0} \in \Omega_{\Upsilon}^{\mathrm{III}}$.

Отсутствие общих элементов у множеств $\Omega^{\mathrm{I}}, \Omega_{\Upsilon}^{\mathrm{II}}, \Omega_{\Upsilon}^{\mathrm{III}}$ очевидно.

СлЕДСТвИЕ. Из соотношений (13)-(15) и представлений (7) функиий $\varphi_{k}(x, \lambda), k=\overline{1, m}$, получаем следующие утверждения.

а) Если $\lambda_{n}=\lambda_{n}^{\mathrm{I}} \in \Omega^{\mathrm{I}}$, то простому собственному значению $\lambda_{n}^{\mathrm{I}}$ соответствует имеющая представление (13) собственная функиия

$$
\widehat{\varphi}\left(x, \lambda_{n}^{\mathrm{I}}, \beta_{n}^{\mathrm{I}}\right)=\frac{1}{\beta_{n}^{\mathrm{I}}} \varphi\left(x, \lambda_{n}^{\mathrm{I}}\right),
$$

где

$$
\begin{gathered}
\beta_{n}^{\mathrm{I}}=\frac{1}{v\left(\pi / 2, \lambda_{n}^{\mathrm{I}}\right)} \prod_{i=1}^{m-1} u_{i}\left(\frac{\pi}{2}, \lambda_{n}^{\mathrm{I}}\right), \\
\varphi(x, \lambda)=\sum_{k=1}^{m-1} \prod_{i=1, i \neq k}^{m-1} u_{i}\left(\frac{\pi}{2}, \lambda\right) \varphi_{k}(x, \lambda) \\
-(m-2) \prod_{i=1}^{m-1} \frac{u_{i}(\pi / 2, \lambda)}{\delta_{1}(\lambda)}\left(v^{\prime}\left(\frac{\pi}{2}, \lambda\right) \varphi_{1}(x, \lambda)-u^{\prime}\left(\frac{\pi}{2}, \lambda\right) \varphi_{m}(x, \lambda)\right),
\end{gathered}
$$

$\delta_{1}(\lambda)=v^{\prime}(\pi / 2, \lambda) u_{1}(\pi / 2, \lambda)-u_{1}^{\prime}(\pi / 2, \lambda) v(\pi / 2, \lambda)$. Аналогично при $\lambda_{n}=\lambda_{n}^{\mathrm{II}} \in \Omega_{2}^{\mathrm{II}}$ u $\lambda_{n}=\lambda_{n}^{\mathrm{III}} \in \Omega_{1}^{\mathrm{III}}$. Hапример, при $\lambda_{n}=\lambda_{n}^{\mathrm{III}} \in \Omega_{1}^{\mathrm{III}}$ простому собственному значению $\lambda_{n}^{\mathrm{III}}$ соответствует имеющая представление $(15)$ (при $\Upsilon=1$ ) собственная функиия

$$
\widehat{\varphi}\left(x, \lambda_{n}^{\mathrm{III}}, \beta_{n}^{\mathrm{III}}\right)=\frac{1}{\beta_{n}^{\mathrm{III}}} \varphi\left(x, \lambda_{n}^{\mathrm{III}}\right)
$$

где

$$
\beta_{n}^{\mathrm{III}}=\frac{u_{m_{1}}^{\prime}\left(\pi / 2, \lambda_{n}^{\mathrm{III}}\right)}{v^{\prime}\left(\pi / 2, \lambda_{n}^{\mathrm{III}}\right)} \prod_{i=1, i \neq m_{1}}^{m-1} u_{i}\left(\frac{\pi}{2}, \lambda_{n}^{\mathrm{III}}\right)
$$

(индекс $m_{1}$ определяется условием $\left.u_{m_{1}}\left(\pi / 2, \lambda_{n}^{\mathrm{III}}\right)=0\right)$. 
б) Если $\lambda_{n}=\lambda_{n}^{\mathrm{III}} \in \Omega_{\Upsilon}^{\mathrm{III}}$, то кратному собственному значению $\lambda_{n}^{\mathrm{III}}$ coответствуют $\Upsilon$ имеющих представление (15) собственных функций

$$
\begin{aligned}
\widehat{\varphi}_{k}\left(x, \lambda_{n}^{\mathrm{III}}\right)= & \frac{1}{u_{m_{1}}^{\prime}\left(\pi / 2, \lambda_{n}^{\mathrm{III}}\right)}\left(u_{m_{k}}^{\prime}\left(\frac{\pi}{2}, \lambda_{n}^{\mathrm{III}}\right) \varphi_{m_{1}}\left(x, \lambda_{n}^{\mathrm{III}}\right)\right. \\
& \left.\quad-u_{m_{1}}^{\prime}\left(\frac{\pi}{2}, \lambda_{n}^{\mathrm{III}}\right) \varphi_{m_{k}}\left(x, \lambda_{n}^{\mathrm{III}}\right)\right), \quad k=\overline{1, \Upsilon-1}, \\
\widehat{\varphi} \Upsilon\left(x, \lambda_{n}^{\mathrm{III}}\right)= & \varphi_{m}\left(x, \lambda_{n}^{\mathrm{III}}\right) .
\end{aligned}
$$

Процесс ортогонализации Сонина-Шмидта [5; гл. 2, § 3, п. 2.3.4] для функций $\widehat{\varphi}_{k}\left(x, \lambda_{n}^{\mathrm{III}}\right)$ приводит $к$ системе взаимно ортогональных собственньх функиий $\chi_{k}\left(x, \lambda_{n}^{\mathrm{III}}\right), k=\overline{1, \Upsilon}$, əде

$$
\begin{gathered}
\chi_{1}\left(x, \lambda_{n}^{\mathrm{III}}\right)=\widehat{\varphi}_{1}\left(x, \lambda_{n}^{\mathrm{III}}\right), \\
\chi_{k}\left(x, \lambda_{n}^{\mathrm{III}}\right)=-\sum_{i=1}^{k-1} \theta_{k i} \chi_{i}\left(x, \lambda_{n}^{\mathrm{III}}\right)+\widehat{\varphi}_{k}\left(x, \lambda_{n}^{\mathrm{III}}\right), \quad k=\overline{2, \Upsilon} \\
\theta_{k i}=\frac{\int_{\Gamma} \widehat{\varphi}_{k}\left(x, \lambda_{n}^{\mathrm{III}}\right) \chi_{i}\left(x, \lambda_{n}^{\mathrm{III}}\right) d x}{\int_{\Gamma} \chi_{i}^{2}\left(x, \lambda_{n}^{\mathrm{III}}\right) d x} .
\end{gathered}
$$

Эти функции таковъ:

$$
\begin{aligned}
& \chi_{k}\left(x, \lambda_{n}^{\mathrm{III}}\right)=\left\{\begin{array}{c}
\frac{\prod_{i=1}^{k} \vartheta_{m_{i}}\left(\lambda_{n}^{\mathrm{III}}\right)}{d_{k}\left(\lambda_{n}^{\mathrm{III}}\right) \vartheta_{m_{j}}\left(\lambda_{n}^{\mathrm{III}}\right)} u_{m_{k+1}}^{\prime}\left(\frac{\pi}{2}, \lambda_{n}^{\mathrm{III}}\right) u_{m_{j}}^{\prime}\left(\frac{\pi}{2}, \lambda_{n}^{\mathrm{III}}\right) u_{m_{j}}\left(x, \lambda_{n}^{\mathrm{III}}\right), \\
x \in \gamma_{m_{j}}, j=\overline{1, k}, \\
-u_{m_{k+1}}\left(x, \lambda_{n}^{\mathrm{III}}\right), \quad x \in \gamma_{m_{k+1}}, \\
0, \quad x \in \gamma_{j}, j=\overline{1, m}, j \neq m_{i}, i=\overline{1, k+1},
\end{array}\right. \\
& \chi_{\Upsilon}\left(x, \lambda_{n}^{\mathrm{III}}\right)=\left\{\begin{array}{c}
\prod_{i=1}^{\Upsilon} \vartheta_{m_{i}}\left(\lambda_{n}^{\mathrm{III}}\right) \\
\overline{d_{m_{0}}\left(\lambda_{n}^{\mathrm{III}}\right) \vartheta_{m_{j}}\left(\lambda_{n}^{\mathrm{III}}\right)} v^{\prime}\left(\frac{\pi}{2}, \lambda_{n}^{\mathrm{III}}\right) u_{m_{j}}^{\prime}\left(\frac{\pi}{2}, \lambda_{n}^{\mathrm{III}}\right) u_{m_{j}}\left(x, \lambda_{n}^{\mathrm{III}}\right), \\
0, \quad x \in \gamma_{m_{j}}, j=\overline{1, \Upsilon}, \quad j=\overline{1, m-1}, j \neq m_{i}, i=\overline{1, \Upsilon} \\
v\left(x, \lambda_{n}^{\mathrm{III}}\right), \quad x \in \gamma_{m},
\end{array}\right.
\end{aligned}
$$

здесь

$$
d_{k}\left(\lambda_{n}^{\mathrm{III}}\right)=\sum_{j=1}^{k} u_{m_{j}}^{\prime 2}\left(\frac{\pi}{2}, \lambda_{n}^{\mathrm{III}}\right) \prod_{i=1, i \neq j}^{k} \vartheta_{m_{i}}\left(\lambda_{n}^{\mathrm{III}}\right), \quad k=\overline{1, \Upsilon}
$$

представления $\vartheta_{j}(\lambda), \vartheta(\lambda)$ указаны ниже перед теоремой 5.

в) Если $\lambda_{n}=\lambda_{n}^{\mathrm{II}} \in \Omega_{\Upsilon}^{\mathrm{II}}$, то кратному собственному значению $\lambda_{n}^{\mathrm{II}}$ соответствуют $\Upsilon-1$ имеющих представление (14) собственных функиий $\widehat{\varphi}_{k}\left(x, \lambda_{n}^{\mathrm{II}}\right)$, $k=\overline{1, \Upsilon-1} ;$ соответствующая система взаимно ортогональных собственных функиий $-\chi_{k}\left(x, \lambda_{n}^{\mathrm{II}}\right), k=\overline{1, \Upsilon-1}$. 
Пусть

$$
\begin{gathered}
\widehat{\omega}_{n}^{\mathrm{I}}=\int_{\Gamma} \widehat{\varphi}^{2}\left(x, \lambda_{n}^{\mathrm{I}}, \beta_{n}^{\mathrm{I}}\right) d x, \quad \widehat{\omega}_{n}^{\mathrm{III}}=\int_{\Gamma} \widehat{\varphi}^{2}\left(x, \lambda_{n}^{\mathrm{III}}, \beta_{n}^{\mathrm{III}}\right) d x \\
\vartheta_{k}(\lambda)=\int_{\gamma_{k}} u_{k}^{2}(x, \lambda) d x, \quad k=\overline{1, m+1}, \quad \vartheta(\lambda)=\int_{\gamma_{m}} v^{2}(x, \lambda) d x .
\end{gathered}
$$

Теорема 5. Пусть $\lambda_{n}$ - собственное значение краевой задачи (1)-(3). Тогда

a) $n p u \lambda_{n}=\lambda_{n}^{\mathrm{I}} \in \Omega^{\mathrm{I}}$

$$
\frac{d}{d \lambda} D\left(\lambda_{n}^{\mathrm{I}}\right)=-\beta_{n}^{\mathrm{I}} \widehat{\omega}_{n}^{\mathrm{I}}, \quad \beta_{n}^{\mathrm{I}}=\frac{1}{\left(\pi / 2, \lambda_{n}^{\mathrm{I}}\right)} \prod_{i=1}^{m-1} u_{i}\left(\frac{\pi}{2}, \lambda_{n}^{\mathrm{I}}\right)
$$

npu $\lambda_{n}=\lambda_{n}^{\mathrm{III}} \in \Omega_{1}^{\mathrm{III}}$

$$
\frac{d}{d \lambda} D\left(\lambda_{n}^{\mathrm{III}}\right)=-\beta_{n}^{\mathrm{III}} \widehat{\omega}_{n}^{\mathrm{III}}, \quad \beta_{n}^{\mathrm{III}}=\frac{u_{m_{1}}^{\prime}\left(\pi / 2, \lambda_{n}^{\mathrm{III}}\right)}{v^{\prime}\left(\pi / 2, \lambda_{n}^{\mathrm{III}}\right)} \prod_{i=1, i \neq m_{1}}^{m-1} u_{i}\left(\frac{\pi}{2}, \lambda_{n}^{\mathrm{III}}\right)
$$

б) при $\lambda_{n}=\lambda_{n}^{\mathrm{II}} \in \Omega_{\Upsilon}^{\mathrm{II}}, 2 \leqslant \Upsilon \leqslant m-1$,

$$
\frac{d}{d \lambda} u_{m_{k}}\left(\frac{\pi}{2}, \lambda_{n}^{\mathrm{II}}\right)=\frac{\vartheta_{m_{k}}\left(\lambda_{n}^{\mathrm{II}}\right)}{u_{m_{k}}^{\prime}\left(\pi / 2, \lambda_{n}^{\mathrm{II}}\right)}, \quad k=\overline{1, \Upsilon}
$$

в) $n$ pu $\lambda_{n}=\lambda_{n}^{\mathrm{III}} \in \Omega_{\Upsilon}^{\mathrm{III}}, 2 \leqslant \Upsilon \leqslant m-1$,

$$
\begin{aligned}
\frac{d}{d \lambda} u_{m_{k}}\left(\frac{\pi}{2}, \lambda_{n}^{\mathrm{III}}\right) & =\frac{\vartheta_{m_{k}}\left(\lambda_{n}^{\mathrm{III}}\right)}{u_{m_{k}}^{\prime}\left(\pi / 2, \lambda_{n}^{\mathrm{III}}\right)}, \quad k=\overline{1, \Upsilon}, \\
\frac{d}{d \lambda} v\left(\frac{\pi}{2}, \lambda_{n}^{\mathrm{III}}\right) & =-\frac{\vartheta\left(\lambda_{n}^{\mathrm{III}}\right)}{v^{\prime}\left(\pi / 2, \lambda_{n}^{\mathrm{III}}\right)} .
\end{aligned}
$$

ДокАзАтельство. а) Пусть $\lambda_{n}=\lambda_{n}^{\mathrm{I}} \in \Omega^{\mathrm{I}}$. Рассмотрим функцию $\varphi(x, \lambda)$ (следствие а) теоремы 4). Применяя формулу Лагранжа (10) к функциям $\varphi(x, \lambda)$ и $\widehat{\varphi}\left(x, \lambda_{n}^{\mathrm{I}}, \beta_{n}^{\mathrm{I}}\right)$, получаем

$$
\left(\lambda-\lambda_{n}^{\prime}\right) \int_{\Gamma} \varphi(x, \lambda) \widehat{\varphi}\left(x, \lambda_{n}^{I}, \beta_{n}^{I}\right) d x=\left\langle\varphi(x, \lambda), \widehat{\varphi}\left(x, \lambda_{n}^{I}, \beta_{n}^{I}\right)\right\rangle_{x=\pi}
$$

(слагаемые в точках $0 \in \gamma_{k}, k=\overline{1, m-1}$, и узле $\xi$ равны нулю в силу представлений функций $\varphi(x, \lambda), \widehat{\varphi}\left(x, \lambda_{n}^{\mathrm{I}}, \beta_{n}^{\mathrm{I}}\right)$ и условий согласования $\left.(2)\right)$. Исходя из представления функции $\widehat{\varphi}\left(x, \lambda_{n}^{\mathrm{I}}, \beta_{n}^{\mathrm{I}}\right)$ на $\gamma_{m}$ можно заменить $\lambda_{n}^{\mathrm{I}}$ в правой части соотношения (17) на $\lambda$ и получить следующую цепочку равенств:

$$
\begin{aligned}
(\lambda- & \left.\lambda_{n}^{\mathrm{I}}\right) \int_{\Gamma} \varphi(x, \lambda) \widehat{\varphi}\left(x, \lambda_{n}^{\mathrm{I}}, \beta_{n}^{\mathrm{I}}\right) d x \\
& =\left[-\varphi^{\prime}(x, \lambda) \widehat{\varphi}\left(x, \lambda, \beta_{n}^{\mathrm{I}}\right)+\varphi(x, \lambda) \widehat{\varphi}^{\prime}\left(x, \lambda, \beta_{n}^{\mathrm{I}}\right)\right]_{x=\pi} \\
& =\left[-\varphi^{\prime}(x, \lambda) \widehat{\varphi}\left(x, \lambda, \beta_{n}^{\mathrm{I}}\right)+\varphi(x, \lambda) \widehat{\varphi}^{\prime}\left(x, \lambda, \beta_{n}^{\mathrm{I}}\right)\right]_{x=\pi / 2 \in \gamma_{m}}=-D(\lambda) .
\end{aligned}
$$

Поделив левые и правые части этих равенств на $\lambda-\lambda_{n}^{\mathrm{I}}$ и переходя к пределу при $\lambda \rightarrow \lambda_{n}^{\mathrm{I}}$, получаем

$$
\frac{d}{d \lambda} D\left(\lambda_{n}^{\mathrm{I}}\right)=-\beta_{n}^{\mathrm{I}} \widehat{\omega}_{n}^{\mathrm{I}} \neq 0 .
$$


Аналогично поступаем и при $\lambda_{n}=\lambda_{n}^{\mathrm{III}} \in \Omega_{1}^{\mathrm{III}}$, применяя формулу Лагранжа к функциям $\varphi(x, \lambda)$ и $\widehat{\varphi}\left(x, \lambda_{n}^{\mathrm{III}}, \beta_{n}^{\mathrm{III}}\right)$.

б) При $\lambda_{n}=\lambda_{n}^{\mathrm{II}} \in \Omega_{\Upsilon}^{\mathrm{II}}, 2 \leqslant \Upsilon \leqslant m-1$, применяя формулу Лагранжа к функциям $\Phi_{k}(x, \lambda)=u_{m_{k}}^{\prime}(\pi / 2, \lambda) \varphi_{m_{1}}(x, \lambda)-u_{m_{1}}^{\prime}(\pi / 2, \lambda) \varphi_{m_{k}}(x, \lambda), k=\overline{2, \Upsilon}$, $\Phi_{k}\left(x, \lambda_{n}^{\mathrm{II}}\right)$, получаем

$$
\begin{aligned}
u_{m_{k}}^{\prime} & \left(\frac{\pi}{2}, \lambda_{n}^{\mathrm{II}}\right) \frac{d}{d \lambda} u_{m_{1}}\left(\frac{\pi}{2}, \lambda_{n}^{\mathrm{II}}\right)+u_{m_{1}}^{\prime}\left(\frac{\pi}{2}, \lambda_{n}^{\mathrm{II}}\right) \frac{d}{d \lambda} u_{m_{k}}\left(\frac{\pi}{2}, \lambda_{n}^{\mathrm{II}}\right) \\
\quad= & \frac{u_{m_{k}}^{\prime}\left(\pi / 2, \lambda_{n}^{\mathrm{II}}\right)}{u_{m_{1}}^{\prime}\left(\pi / 2, \lambda_{n}^{\mathrm{II}}\right)} \vartheta_{m_{1}}\left(\lambda_{n}^{\mathrm{II}}\right)+\frac{u_{m_{1}}^{\prime}\left(\pi / 2, \lambda_{n}^{\mathrm{II}}\right)}{u_{m_{k}}^{\prime}\left(\pi / 2, \lambda_{n}^{\mathrm{II}}\right)} \vartheta_{m_{k}}\left(\lambda_{n}^{\mathrm{II}}\right), \quad k=\overline{2, \Upsilon} .
\end{aligned}
$$

Далее для функций $\Phi(x, \lambda)\left(\right.$ см. пункт в)) и $\Phi\left(x, \lambda_{n}^{\mathrm{II}}\right)$ выводим следующую цепочку равенств:

$$
\begin{aligned}
u_{m_{1}}^{\prime}\left(\frac{\pi}{2}, \lambda_{n}^{\mathrm{II}}\right)\left(\sum_{k=2}^{\Upsilon} u_{m_{k}}^{\prime}\left(\frac{\pi}{2}, \lambda_{n}^{\mathrm{II}}\right)\right)^{2} \frac{d}{d \lambda} u_{m_{1}}\left(\frac{\pi}{2}, \lambda_{n}^{\mathrm{II}}\right) \\
+u_{m_{1}}^{\prime 2}\left(\frac{\pi}{2}, \lambda_{n}^{\mathrm{II}}\right) \sum_{k=2}^{\Upsilon} u_{m_{k}}^{\prime}\left(\frac{\pi}{2}, \lambda_{n}^{\mathrm{II}}\right) \frac{d}{d \lambda} u_{m_{k}}\left(\frac{\pi}{2}, \lambda_{n}^{\mathrm{II}}\right) \\
=\left(\sum_{k=2}^{\Upsilon} u_{m_{k}}^{\prime}\left(\frac{\pi}{2}, \lambda_{n}^{\mathrm{II}}\right)\right)^{2} \vartheta_{m_{1}}\left(\lambda_{n}^{\mathrm{II}}\right)+u_{m_{1}}^{\prime 2}\left(\frac{\pi}{2}, \lambda_{n}^{\mathrm{II}}\right) \sum_{k=2}^{\Upsilon} \vartheta_{m_{k}}\left(\lambda_{n}^{\mathrm{II}}\right) .
\end{aligned}
$$

Соотношения (18), (19) задают систему $\Upsilon$ уравнений, однозначно разрешенную относительно $\frac{d}{d \lambda} u_{m_{k}}\left(\pi / 2, \lambda_{n}^{\mathrm{II}}\right), k=\overline{1, \Upsilon}$, и аналогичную системе (18), (19). Ее решение имеет вид

$$
\frac{d}{d \lambda} u_{m_{k}}\left(\frac{\pi}{2}, \lambda_{n}^{\mathrm{II}}\right)=\frac{\vartheta_{m_{k}}\left(\lambda_{n}^{\mathrm{II}}\right)}{u_{m_{k}}^{\prime}\left(\pi / 2, \lambda_{n}^{\mathrm{II}}\right)} \neq 0, \quad k=\overline{1, \Upsilon}
$$

в) Пусть $\lambda_{n}=\lambda_{n}^{\mathrm{III}} \in \Omega_{\Upsilon}^{\mathrm{III}}, 2 \leqslant \Upsilon \leqslant m-1$. Очевидно, что функции $\varphi_{k}\left(x, \lambda_{n}^{\mathrm{III}}\right)$ (представление $(7))$ являются собственными функциями для $\lambda_{n}^{\mathrm{III}}$. Применяя формулу Лагранжа $(10)$ к функциям $\varphi_{m_{k}}(x, \lambda), \varphi_{m_{k}}\left(x, \lambda_{n}^{\mathrm{III}}\right)$, получим

$$
\begin{aligned}
(\lambda- & \left.\lambda_{n}^{\mathrm{III}}\right) \int_{\Gamma} \varphi_{m_{k}}(x, \lambda) \widehat{\varphi}_{m_{k}}\left(x, \lambda_{n}^{\mathrm{III}}\right) d x=\left\langle\varphi_{m_{k}}(x, \lambda), \widehat{\varphi}_{m_{k}}\left(x, \lambda_{n}^{\mathrm{III}}\right)\right\rangle_{x=\pi} \\
= & \frac{u_{m_{k}}^{\prime}\left(\pi / 2, \lambda_{n}^{\mathrm{III}}\right)}{v^{\prime}\left(\pi / 2, \lambda_{n}^{\mathrm{III}}\right)}\left[v^{\prime}\left(\frac{\pi}{2}, \lambda\right) u_{m_{k}}\left(\frac{\pi}{2}, \lambda\right)-v\left(\frac{\pi}{2}, \lambda\right) u_{m_{k}}^{\prime}\left(\frac{\pi}{2}, \lambda\right)\right]
\end{aligned}
$$

(здесь, как и выше, возможна замена $\lambda_{n}^{\mathrm{III}}$ на $\lambda$ ). Переход к пределу при $\lambda \rightarrow \lambda_{n}^{\mathrm{III}}$ дает

$$
\begin{aligned}
v^{\prime}\left(\frac{\pi}{2}, \lambda_{n}^{\mathrm{III}}\right) \frac{d}{d \lambda} u_{m_{k}}\left(\frac{\pi}{2}, \lambda_{n}^{\mathrm{III}}\right)-u_{m_{k}}^{\prime}\left(\frac{\pi}{2}, \lambda_{n}^{\mathrm{III}}\right) \frac{d}{d \lambda} v\left(\frac{\pi}{2}, \lambda_{n}^{\mathrm{III}}\right) \\
=\frac{v^{\prime}\left(\pi / 2, \lambda_{n}^{\mathrm{III}}\right)}{u_{m_{k}}^{\prime}\left(\pi / 2, \lambda_{n}^{\mathrm{III}}\right)} \vartheta_{m_{k}}\left(\lambda_{n}^{\mathrm{III}}\right)+\frac{u_{m_{k}}^{\prime}\left(\pi / 2, \lambda_{n}^{\mathrm{III}}\right)}{v^{\prime}\left(\pi / 2, \lambda_{n}^{\mathrm{II}}\right)} \vartheta\left(\lambda_{n}^{\mathrm{III}}\right), \quad k=\overline{1, \Upsilon .}
\end{aligned}
$$

Рассмотрим функцию

$$
\Phi(x, \lambda)=\sum_{k=2}^{\Upsilon}\left(u_{m_{k}}^{\prime}\left(\frac{\pi}{2}, \lambda\right) \varphi_{m_{1}}(x, \lambda)-u_{m_{1}}^{\prime}\left(\frac{\pi}{2}, \lambda\right) \varphi_{m_{k}}(x, \lambda)\right) .
$$


Тогда $\Phi\left(x, \lambda_{n}^{\mathrm{III}}\right)-$ собственная функция. Применяя формулу Лагранжа для $\Phi(x, \lambda)$ и $\Phi\left(x, \lambda_{n}^{\mathrm{III}}\right)$, получаем

$$
\begin{aligned}
u_{m_{1}}^{\prime}\left(\frac{\pi}{2}, \lambda_{n}^{\mathrm{III}}\right)\left(\sum_{k=2}^{\Upsilon} u_{m_{k}}^{\prime}\left(\frac{\pi}{2}, \lambda_{n}^{\mathrm{III}}\right)\right)^{2} \frac{d}{d \lambda} u_{m_{1}}\left(\frac{\pi}{2}, \lambda_{n}^{\mathrm{III}}\right) \\
+u_{m_{1}}^{\prime 2}\left(\frac{\pi}{2}, \lambda_{n}^{\mathrm{III}}\right) \sum_{k=2}^{\Upsilon} u_{m_{k}}^{\prime}\left(\frac{\pi}{2}, \lambda_{n}^{\mathrm{III}}\right) \frac{d}{d \lambda} u_{m_{k}}\left(\frac{\pi}{2}, \lambda_{n}^{\mathrm{III}}\right) \\
=\left(\sum_{k=2}^{\Upsilon} u_{m_{k}}^{\prime}\left(\frac{\pi}{2}, \lambda_{n}^{\mathrm{III}}\right)\right)^{2} \vartheta_{m_{1}}\left(\lambda_{n}^{\mathrm{III}}\right)+{u^{\prime}}_{m_{1}}^{2}\left(\frac{\pi}{2}, \lambda_{n}^{\mathrm{III}}\right) \sum_{k=2}^{\Upsilon} \vartheta_{m_{k}}\left(\lambda_{n}^{\mathrm{III}}\right) .
\end{aligned}
$$

Таким образом, получена система (20), (21), состоящая из $\Upsilon+1$ уравнения относительно

$$
\frac{d}{d \lambda} u_{m_{k}}\left(\frac{\pi}{2}, \lambda_{n}^{\mathrm{III}}\right), \quad k=\overline{1, \Upsilon}, \quad \frac{d}{d \lambda} v\left(\frac{\pi}{2}, \lambda_{n}^{\mathrm{III}}\right)
$$

с определителем

$u_{m_{1}}^{\prime 2}\left(\frac{\pi}{2}, \lambda_{n}^{\mathrm{III}}\right)\left(v^{\prime}\left(\frac{\pi}{2}, \lambda_{n}^{\mathrm{III}}\right)\right)^{\Upsilon-1}\left(\left(\sum_{k=2}^{\Upsilon} u_{m_{k}}^{\prime}\left(\frac{\pi}{2}, \lambda_{n}^{\mathrm{III}}\right)\right)^{2}+\sum_{k=2}^{\Upsilon} u_{m_{k}}^{\prime 2}\left(\frac{\pi}{2}, \lambda_{n}^{\mathrm{III}}\right)\right) \neq 0$.

Значит, система (20), (21) имеет единственное решение

$$
\begin{aligned}
\frac{d}{d \lambda} u_{m_{k}}\left(\frac{\pi}{2}, \lambda_{n}^{\mathrm{III}}\right) & =\frac{\vartheta_{m_{k}}\left(\lambda_{n}^{\mathrm{III}}\right)}{u_{m_{k}}^{\prime}\left(\pi / 2, \lambda_{n}^{\mathrm{III}}\right)} \neq 0, \quad k=\overline{1, \Upsilon}, \\
\frac{d}{d \lambda} v\left(\frac{\pi}{2}, \lambda_{n}^{\mathrm{III}}\right) & =-\frac{\vartheta\left(\lambda_{n}^{\mathrm{III}}\right)}{v^{\prime}\left(\pi / 2, \lambda_{n}^{\mathrm{III}}\right)} \neq 0 .
\end{aligned}
$$

СледСТвиЕ. Отличие от нуля производных

$$
\begin{gathered}
\frac{d}{d \lambda} D\left(\lambda_{n}^{\mathrm{I}}\right), \quad \frac{d}{d \lambda} D\left(\lambda_{n}^{\mathrm{III}}\right), \\
\frac{d}{d \lambda} u_{m_{k}}\left(\frac{\pi}{2}, \lambda_{n}^{\mathrm{II}}\right), \quad \frac{d}{d \lambda} v\left(\frac{\pi}{2}, \lambda_{n}^{\mathrm{III}}\right), \quad \frac{d}{d \lambda} u_{m_{k}}\left(\frac{\pi}{2}, \lambda_{n}^{\mathrm{III}}\right)
\end{gathered}
$$

означает простоту нулей $\lambda_{n}^{\mathrm{I}}, \lambda_{n}^{\mathrm{II}}, \lambda_{n}^{\mathrm{III}}$ функции $D(\lambda)$.

Функиией Грина краевой задачи (1)-(3) [3; гл. 3, § 3.2, п. 3.2.3] назовем функцию $G(x, t, \lambda)$ такую, что решение неоднородной задачи

$$
\begin{gathered}
L y-\lambda y=f(x), \\
y^{\prime}(0)_{\gamma_{k}}-h_{k} y(0)_{\gamma_{k}}=0, \quad k=\overline{1, m-1}, \quad y^{\prime}(\pi)_{\gamma_{m}}+H y(\pi)_{\gamma_{m}}=0
\end{gathered}
$$

при любой функции $f(x)$ может быть представлено в виде

$$
y(x, \lambda)=\int_{\Gamma} G(x, t, \lambda) f(t) d t,
$$

т.е. функция $y(x, \lambda)$ является истокопредставимой с помощью ядра $G(x, t, \lambda)$.

Пусть

$$
\begin{aligned}
\Delta_{k}(\lambda) & =\left\langle\varphi_{k}(x, \lambda), \varphi_{m}(x, \lambda)\right\rangle_{\gamma_{k}}, \quad k=\overline{1, m-1} \\
\Delta_{m}(\lambda) & =\left\langle\varphi_{1}(x, \lambda), \varphi_{m}(x, \lambda)\right\rangle_{\gamma_{m}} .
\end{aligned}
$$


В соответствии с представлениями (7) функций $\varphi_{k}(x, \lambda)$ имеем

$$
\Delta_{1}(\lambda)=\Delta_{m}(\lambda)=\delta_{1}(\lambda), \quad \Delta_{k}(\lambda)=-v\left(\frac{\pi}{2}, \lambda\right) u_{k}^{\prime}\left(\frac{\pi}{2}, \lambda\right), \quad k=\overline{2, m-1} .
$$

Определим $G(x, t, \lambda)$ соотношением $G(x, t, \lambda)=G_{0}(x, t, \lambda)+g(x, \lambda)$, где

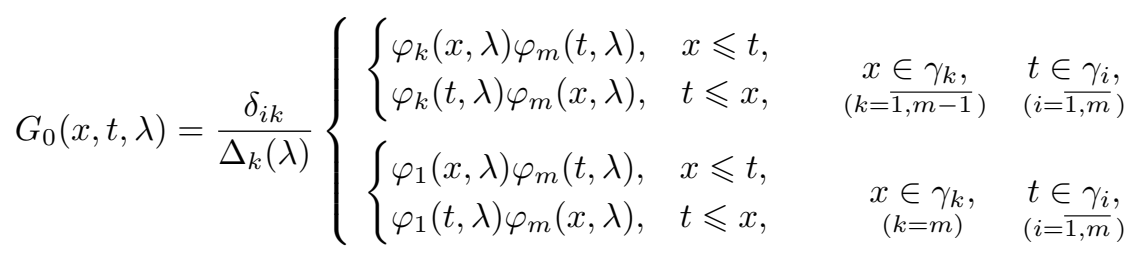

$$
\begin{aligned}
& g(x, \lambda)_{\gamma_{k}}=c_{k} \varphi_{k}(x, \lambda), \quad k=\overline{1, m},
\end{aligned}
$$

$c_{k}, k=\overline{1, m}$, определяются как решение системы $m$ линейных алгебраических уравнений

$$
\left\{\begin{array}{l}
u_{k}\left(\frac{\pi}{2}, \lambda\right) c_{k}-v\left(\frac{\pi}{2}, \lambda\right) c_{m} \\
\quad=G_{0}(x, t, \lambda)_{x=\pi / 2 \in \gamma_{m}}-G_{0}(x, t, \lambda)_{x=\pi / 2 \in \gamma_{k}}, \quad k=\overline{1, m-1} \\
\sum_{k=1}^{m-1} u_{k}^{\prime}\left(\frac{\pi}{2}, \lambda\right) c_{k}-v^{\prime}\left(\frac{\pi}{2}, \lambda\right) c_{m} \\
\quad=\frac{\partial G_{0}}{\partial x}(x, t, \lambda)_{x=\pi / 2 \in \gamma_{m}}-\sum_{k=1}^{m-1} \frac{\partial G_{0}}{\partial x}(x, t, \lambda)_{x=\pi / 2 \in \gamma_{k}}
\end{array}\right.
$$

порожденной непрерывностью решения $y(x, \lambda)$ в узле $\xi$ и условиями согласования (2). Определитель системы равен

$$
D(\lambda)=v\left(\frac{\pi}{2}, \lambda\right) \sum_{k=1}^{m-1}\left\{u_{k}^{\prime}\left(\frac{\pi}{2}, \lambda\right) \prod_{\substack{i=1 \\ i \neq k)}}^{m-1} u_{i}\left(\frac{\pi}{2}, \lambda\right)\right\}-v^{\prime}\left(\frac{\pi}{2}, \lambda\right) \prod_{i=1}^{m-1} u_{i}\left(\frac{\pi}{2}, \lambda\right)
$$

коэффициенты $c_{k} \equiv c_{k}(t, \lambda), t \in \Gamma, k=\overline{1, m}$, определяются следующими соотношениями:

$$
\begin{gathered}
c_{k}(t, \lambda)_{\gamma_{k}}=-\frac{v(\pi / 2, \lambda) D_{k}(\lambda)}{\Delta_{k}(\lambda) D(\lambda)} \varphi_{k}(t, \lambda), \quad k \neq m, \\
c_{m}(t, \lambda)_{\gamma_{m}}=-\frac{D_{k}(\lambda)}{\Delta_{m}(\lambda) D(\lambda)} \varphi_{m}(t, \lambda), \\
c_{i}(t, \lambda)_{\gamma_{k}}=-\frac{v(\pi / 2, \lambda)}{D(\lambda)} \prod_{\substack{j=1 \\
j \neq i, k)}}^{m-1} u_{j}\left(\frac{\pi}{2}, \lambda\right) \varphi_{k}(t, \lambda), \quad i=\overline{1, m-1,} \quad i \neq k, \quad k \neq m, \\
c_{m}(t, \lambda)_{\gamma_{k}}=c_{k}(t, \lambda)_{\gamma_{m}}=-\frac{1}{D(\lambda)} \prod_{\substack{j=1 \\
(j \neq k)}}^{m-1} u_{j}\left(\frac{\pi}{2}, \lambda\right) \varphi_{k}(t, \lambda), \quad k
\end{gathered}
$$


здесь

$$
\begin{aligned}
& D_{1}(\lambda)=v\left(\frac{\pi}{2}, \lambda\right) \sum_{j=2}^{m-1}\left\{u_{j}^{\prime}\left(\frac{\pi}{2}, \lambda\right) \prod_{\substack{i=2 \\
(i \neq j)}}^{m-1} u_{i}\left(\frac{\pi}{2}, \lambda\right)\right\} \\
& D_{k}(\lambda)=v\left(\frac{\pi}{2}, \lambda\right) \sum_{\substack{j=1 \\
(j \neq k)}}^{m-1} u_{j}^{\prime}\left(\frac{\pi}{2}, \lambda\right) \prod_{\substack{i=1 \\
i \neq k, j)}}^{m-1} u_{i}\left(\frac{\pi}{2}, \lambda\right)-v^{\prime}\left(\frac{\pi}{2}, \lambda\right) \prod_{\substack{i=1 \\
i \neq k}}^{m-1} u_{i}\left(\frac{\pi}{2}, \lambda\right), \\
& k=\overline{2, m-1}, \\
& D_{m}(\lambda)=u_{1}\left(\frac{\pi}{2}, \lambda\right) \sum_{j=1}^{m-1} u_{j}^{\prime}\left(\frac{\pi}{2}, \lambda\right) \prod_{\substack{i=1 \\
i \neq j}}^{m-1} u_{i}\left(\frac{\pi}{2}, \lambda\right)-u_{1}^{\prime}\left(\frac{\pi}{2}, \lambda\right) \prod_{i=1}^{m-1} u_{i}\left(\frac{\pi}{2}, \lambda\right) .
\end{aligned}
$$

Таким образом, если $\lambda$ не является нулем функций $\Delta_{k}(\lambda) D(\lambda)$, то функция Грина краевой задачи (1)-(3) существует и принимает вид

$$
G(x, t, \lambda)=G_{0}(x, t, \lambda)+c_{k}(t, \lambda) \varphi_{k}(x, \lambda), \quad x \in \gamma_{k}, \quad k=\overline{1, m}
$$

Очевидна симметричность функции $G(x, t, \lambda)$ по переменным $x, t \in \Gamma$.

Изучим асимптотическое поведение функций $\varphi_{k}(x, \lambda), k=\overline{1, m}$, в комплексной $\lambda$-плоскости спектрального параметра. Пусть $\lambda=\rho^{2}, \tau=\operatorname{Im} \rho$, обозначим $G_{\delta}=\{\rho:|\rho-k| \geqslant \delta>0, k=0,1,2, \ldots\}$.

Теорема 6. При $|\rho| \rightarrow+\infty$ вернъ следующие асимптотические формуль:

$$
\begin{aligned}
& \underset{(k=\overline{1, m-1})}{\varphi_{k}(x, \lambda)}= \begin{cases}\cos \left(\frac{\rho \pi}{2}\right) \cos \rho\left(\frac{\pi}{2}-x\right), & \quad x \in \gamma_{i}, \\
\cos \rho x, & \begin{array}{l}
(i=1, m-1 \\
x \in k)
\end{array}+O\left(\frac{\exp (|\tau| x)}{|\rho|}\right),\end{cases} \\
& \varphi_{m}(x, \lambda)=\rho \begin{cases}\cos \left(\frac{\rho \pi}{2}\right) \cos \rho\left(\frac{\pi}{2}-x\right), & \begin{array}{l}
x \in \gamma_{i}, \\
(i=2, m-1
\end{array},+O\left(\frac{\exp (|\tau| x)}{|\rho|}\right), \\
\cos \rho(\pi-x), & x \in \gamma_{1} \cup \gamma_{m},\end{cases} \\
& \underset{(k=1, m-1)}{\varphi_{k}^{\prime}(x, \lambda)}=\rho \begin{cases}\cos \left(\frac{\rho \pi}{2}\right) \sin \rho\left(\frac{\pi}{2}-x\right), & \underset{(i=1, m-1, i \neq k)}{x \in \gamma_{i},}+O(\exp (|\tau| x)), \\
-\sin \rho x, & x \in \gamma_{k} \cup \gamma_{m},\end{cases} \\
& \varphi_{m}^{\prime}(x, \lambda)=\rho\left\{\begin{array}{ll}
\cos \left(\frac{\rho \pi}{2}\right) \sin \rho\left(\frac{\pi}{2}-x\right), & x \in \gamma_{i}, \\
\sin \rho(\pi-x), & x \in \gamma_{1} \cup \gamma_{m},
\end{array} \quad+O(\exp (|\tau| x)),\right. \\
& \Delta_{k}(\lambda)=\rho \sin \rho \pi\left\{\begin{array}{ll}
\frac{1}{2}, & k=\overline{2, m-1}, \\
1, & k=1, m,
\end{array}+O(\exp (|\tau| x)), \quad \rho \in G_{\delta} .\right.
\end{aligned}
$$

ДокАЗАтЕЛЬство. Асимптотические формулы (23) можно получить из представлений (7) для функций $\varphi_{k}(x, \lambda), k=\overline{1, m}$, и вида $\Delta_{k}(\lambda)$, используя следующие асимптотические формулы для функций $u_{k}(x, \lambda), v_{k}(x, \lambda), k=\overline{1, m}$, 
и определителя $\delta_{1}(\lambda)[6 ;$ гл. $1, \S 1.1$, лемма 1.1 .2 , теорема 1.1 .3$]:$ при $|\rho| \rightarrow+\infty$

$$
\begin{aligned}
u_{k}(x, \lambda) & =\cos \rho x+O\left(\frac{\exp (|\tau| x)}{|\rho|}\right), \\
u_{k}^{\prime}(x, \lambda) & =-\rho \sin \rho x+O(\exp (|\tau| x)), \\
v_{k}(x, \lambda) & =\cos \rho(\pi-x)+O\left(\frac{\exp (|\tau| x)}{|\rho|}\right), \\
v_{k}^{\prime}(x, \lambda) & =\rho \sin \rho(\pi-x)+O(\exp (|\tau| x)), \\
\delta_{1}(\lambda) & =\rho \sin \rho \pi[1+O(\exp (|\tau| x))]
\end{aligned}
$$

при $\rho \in G_{\delta}$.

СлЕДСТВИЕ. Из представления (22) и асимптотических формул (23) следует, что для функции Грина верна оченка

$$
G(x, t, \lambda) \leqslant \frac{C}{|\rho|}, \quad \rho \in G_{\delta},
$$

при фиксированном $\delta>0(C-$ постоянная $)$.

Теорема 7. Система собственных функиий краевой задачи (1)-(3) полна и образует ортогоналъный базис в $L_{2}(\Gamma)$.

ДоказАтеЛЬство. Рассмотрим истокопредставимую функцию $Y(x, \lambda)$ :

$$
Y(x, \lambda)=\int_{\Gamma} G(x, t, \lambda) f(t) d t, \quad f(x) \in C[\Gamma],
$$

и в комплексной $\lambda$-плоскости спектрального параметра получим вычеты в точ$\operatorname{Kax} \lambda_{n} \in \Omega$.

Используя представление функции Грина (22), на каждом из ребер $\gamma_{k}, k=$ $\overline{1, m}$, получим следующие соотношения.

1) При $\lambda_{n}=\lambda_{n}^{\mathrm{I}} \in \Omega^{\mathrm{I}}$

$$
\begin{aligned}
\underset{\lambda=\lambda_{n}^{\mathrm{I}}}{\operatorname{Res}} Y(x, \lambda)_{\gamma_{k}}=\lim _{\lambda \rightarrow \lambda_{n}^{\mathrm{I}}}\left(\lambda-\lambda_{n}^{\mathrm{I}}\right) Y(x, \lambda)_{\gamma_{k}} & \\
=- & \lim _{\lambda \rightarrow \lambda_{n}^{\mathrm{I}}} \frac{\left(\lambda-\lambda_{n}^{\mathrm{I}}\right)}{D(\lambda)} \prod_{\substack{i=1 \\
(i \neq k)}}^{m-1} u_{i}\left(\frac{\pi}{2}, \lambda\right)\left\{\sum_{j=1}^{m-1} \frac{v(\pi / 2, \lambda)}{u_{j}(\pi / 2, \lambda)} \int_{\gamma_{j}} \varphi_{j}(t, \lambda) f(t) d t\right. \\
& \left.+\int_{\gamma_{m}} \varphi_{m}(t, \lambda) f(t) d t\right\} \varphi_{k}(x, \lambda), \quad k=\overline{1, m-1}
\end{aligned}
$$

$$
\begin{aligned}
\underset{\lambda=\lambda_{n}^{\mathrm{I}}}{\operatorname{Res}} Y(x, \lambda)_{\gamma_{m}}=\lim _{\lambda \rightarrow \lambda_{n}^{\mathrm{I}}}\left(\lambda-\lambda_{n}^{\mathrm{I}}\right) Y(x, \lambda)_{\gamma_{m}} \\
=-\lim _{\lambda \rightarrow \lambda_{n}^{\mathrm{I}}} \frac{\left(\lambda-\lambda_{n}^{\mathrm{I}}\right)}{D(\lambda) v(\pi / 2, \lambda)} \prod_{i=1}^{m-1} u_{i}\left(\frac{\pi}{2}, \lambda\right)\left\{\sum_{j=1}^{m-1} \frac{v(\pi / 2, \lambda)}{u_{j}(\pi / 2, \lambda)} \int_{\gamma_{j}} \varphi_{j}(t, \lambda) f(t) d t\right. \\
\left.\quad+\int_{\gamma_{m}} \varphi_{m}(t, \lambda) f(t) d t\right\} \varphi_{m}(x, \lambda) .
\end{aligned}
$$

Таким образом, учитывая следствие а) из теоремы 4 и п. а) теоремы 5 ,

$$
\operatorname{Res}_{\lambda=\lambda_{n}^{\mathrm{I}}} Y(x, \lambda)_{\Gamma}=\frac{1}{\widehat{\omega}_{n}^{\mathrm{I}}} \widehat{\varphi}\left(x, \lambda_{n}^{\mathrm{I}}, \beta_{n}^{\mathrm{I}}\right) \int_{\Gamma} \widehat{\varphi}\left(t, \lambda_{n}^{\mathrm{I}}, \beta_{n}^{\mathrm{I}}\right) f(t) d t .
$$


Аналогичные рассуждения для $\lambda_{n}=\lambda_{n}^{\mathrm{III}} \in \Omega_{1}^{\mathrm{III}}$ приводят к соотношению

$$
\operatorname{Res}_{\lambda=\lambda_{n}^{\mathrm{III}}} Y(x, \lambda)_{\Gamma}=\frac{1}{\widehat{\omega}_{n}^{\mathrm{III}}} \widehat{\varphi}\left(x, \lambda_{n}^{\mathrm{III}}, \beta_{n}^{\mathrm{III}}\right) \int_{\Gamma} \widehat{\varphi}\left(t, \lambda_{n}^{\mathrm{III}}, \beta_{n}^{\mathrm{III}}\right) f(t) d t .
$$

2) Пусть $\left.\lambda_{n}=\lambda_{n}^{\mathrm{III}} \in \Omega_{\Upsilon}^{\mathrm{III}}, 2 \leqslant \Upsilon \leqslant m-1\right)$. Тогда

$\operatorname{Res}_{\lambda=\lambda_{n}^{\mathrm{III}}} Y(x, \lambda)_{\Gamma}=\lim _{\lambda \rightarrow \lambda_{n}^{\mathrm{III}}}\left(\lambda-\lambda_{n}^{\mathrm{III}}\right) Y(x, \lambda)_{\Gamma}$

$$
\begin{aligned}
& \left\{\begin{array}{l}
\frac{\vartheta\left(\lambda_{n}^{\mathrm{III}}\right) u_{m_{1}}^{\prime}\left(\pi / 2, \lambda_{n}^{\mathrm{III}}\right)}{\vartheta_{m_{1}}\left(\lambda_{n}^{\mathrm{III}}\right) v^{\prime}\left(\pi / 2, \lambda_{n}^{\mathrm{III}}\right)} \varphi_{m_{1}}\left(x, \lambda_{n}^{\mathrm{III}}\right) \\
\quad \times \int_{\Gamma}\left(\sum_{i=1}^{\Upsilon-1} \frac{u_{m_{i+1}}^{\prime}\left(\pi / 2, \lambda_{n}^{\mathrm{III}}\right)}{\vartheta_{m_{i+1}}\left(\lambda_{n}^{\mathrm{III}}\right)} \widehat{\varphi}_{i}\left(t, \lambda_{n}^{\mathrm{III}}\right)+\widehat{\varphi}_{\Upsilon}\left(t, \lambda_{n}^{\mathrm{III}}\right)\right) f(t) d t, \quad x \in \gamma_{m_{1}},
\end{array}\right. \\
& \varphi_{m_{k}}\left(x, \lambda_{n}^{\mathrm{III}}\right)\left(-\frac{\vartheta\left(\lambda_{n}^{\mathrm{III}}\right) u_{m_{k}}^{\prime}\left(\pi / 2, \lambda_{n}^{\mathrm{III}}\right)}{\vartheta_{m_{k}}\left(\lambda_{n}^{\mathrm{III}}\right) v^{\prime}\left(\pi / 2, \lambda_{n}^{\mathrm{III}}\right)}\left(\frac{u_{m_{1}}^{\prime 2}\left(\pi / 2, \lambda_{n}^{\mathrm{III}}\right)}{\vartheta_{m_{1}}\left(\lambda_{n}^{\mathrm{III}}\right) u_{m_{k}}^{\prime}\left(\pi / 2, \lambda_{n}^{\mathrm{III}}\right)}\right.\right. \\
& \times \int_{\Gamma} \widehat{\varphi}_{k-1}\left(t, \lambda_{n}^{\mathrm{III}}\right) f(t) d t+\sum_{\substack{i=1 \\
i \neq k-1)}}^{\Upsilon-1} \frac{u_{m_{i+1}}^{\prime}\left(\pi / 2, \lambda_{n}^{\mathrm{III}}\right)}{\vartheta_{m_{i+1}}\left(\lambda_{n}^{\mathrm{III}}\right)} \\
& \left.\times \int_{\Gamma}\left(\frac{u_{m_{i+1}}^{\prime}\left(\pi / 2, \lambda_{n}^{\mathrm{III}}\right)}{u_{m_{k}}^{\prime}\left(\pi / 2, \lambda_{n}^{\mathrm{III}}\right)} \widehat{\varphi}_{k-1}\left(t, \lambda_{n}^{\mathrm{III}}\right)-\widehat{\varphi}_{i}\left(t, \lambda_{n}^{\mathrm{III}}\right)\right) f(t) d t\right) \\
& \left.-\frac{u_{m_{k}}^{\prime}\left(\pi / 2, \lambda_{n}^{\mathrm{III}}\right)}{\vartheta_{m_{k}}\left(\lambda_{n}^{\mathrm{III}}\right)} \int_{\Gamma}\left(\frac{v^{\prime}\left(\pi / 2, \lambda_{n}^{\mathrm{III}}\right)}{u_{m_{k}}^{\prime}\left(\pi / 2, \lambda_{n}^{\mathrm{III}}\right)} \widehat{\varphi}_{k-1}\left(t, \lambda_{n}^{\mathrm{III}}\right)-\widehat{\varphi}_{\Upsilon}\left(t, \lambda_{n}^{\mathrm{III}}\right)\right) f(t) d t\right), \\
& x \in \gamma_{m_{k}}, \quad k=\overline{2, \Upsilon} \text {, } \\
& 0, \quad x \in \gamma_{k}, \quad k=\overline{1, m-1}, \quad k \neq m_{i}, \quad i=\overline{1, \Upsilon},
\end{aligned}
$$$$
\varphi_{m}\left(x, \lambda_{n}^{\mathrm{III}}\right)\left(\frac{u_{m_{1}}^{\prime 2}\left(\pi / 2, \lambda_{n}^{\mathrm{III}}\right)}{\vartheta_{m_{1}}\left(\lambda_{n}^{\mathrm{III}}\right) v^{\prime}\left(\pi / 2, \lambda_{n}^{\mathrm{III}}\right)} \int_{\Gamma} \widehat{\varphi}_{\Upsilon}\left(t, \lambda_{n}^{\mathrm{III}}\right) f(t) d t\right.
$$$$
+\sum_{i=1}^{\Upsilon-1} \frac{u_{m_{i+1}}^{\prime}\left(\pi / 2, \lambda_{n}^{\mathrm{III}}\right)}{\vartheta_{m_{i+1}}\left(\lambda_{n}^{\mathrm{III}}\right)}
$$$$
\left.\times \int_{\Gamma}\left(\frac{u_{m_{i+1}}^{\prime}\left(\pi / 2, \lambda_{n}^{\mathrm{III}}\right)}{\vartheta\left(\lambda_{n}^{\mathrm{III}}\right)} \widehat{\varphi}_{\Upsilon}\left(t, \lambda_{n}^{\mathrm{III}}\right)-\widehat{\varphi}_{i}\left(t, \lambda_{n}^{\mathrm{III}}\right)\right) f(t) d t\right), \quad x \in \gamma_{m} .
$$

Отсюда, учитывая формулу (16) и п. б) теоремы 5 , получаем

$$
\operatorname{Res}_{\lambda=\lambda_{n}^{\mathrm{III}}} Y(x, \lambda)_{\Gamma}=\sum_{i=1}^{\Upsilon} \frac{1}{\int_{\Gamma} \chi_{i}^{2}\left(t, \lambda_{n}^{\mathrm{III}}\right) d t} \chi_{i}\left(x, \lambda_{n}^{\mathrm{III}}\right) \int_{\Gamma} \chi_{i}\left(t, \lambda_{n}^{\mathrm{III}}\right) f(t) d t .
$$

3) При $\lambda_{n}=\lambda_{n}^{\mathrm{II}} \in \Omega_{\Upsilon}^{\mathrm{II}}, 2 \leqslant \Upsilon \leqslant m-1$, рассуждения, аналогичные предыдущему п. 2), приводят к представлению

$$
\operatorname{Res}_{\lambda=\lambda_{n}^{\mathrm{II}}} Y(x, \lambda)_{\Gamma}=\sum_{i=1}^{\Upsilon-1} \frac{1}{\int_{\Gamma} \chi_{i}^{2}\left(t, \lambda_{n}^{\mathrm{II}}\right) d t} \chi_{i}\left(x, \lambda_{n}^{\mathrm{II}}\right) \int_{\Gamma} \chi_{i}\left(t, \lambda_{n}^{\mathrm{II}}\right) f(t) d t .
$$

Из полученных представлений вычетов (25)-(28) в точках $\lambda_{n}$ следует, что если функция $f(x)$ ортогональна всем собственным функциям, то

$$
\operatorname{Res}_{\lambda=\lambda_{n}} Y(x, \lambda)=0
$$


и, следовательно, при каждом фиксированном $x \in \Gamma$ функция $Y(x, \lambda)$ является целой по $\lambda$. Из (24) при фиксированном $\delta>0$ и достаточно большом $\rho^{*}>0$ получаем [6; гл. $1, \S 1.1$, теорема 1.1 .5$]$ оценку

$$
|Y(x, \lambda)| \leqslant \frac{C_{\delta}}{|\rho|}, \quad \rho \in G_{\delta}, \quad|\rho| \geqslant \rho^{*}
$$

(здесь $C_{\delta}$ - фиксированная постоянная). Используя принцип максимума модуля для аналитических функций [7; гл. IV,$\S 11$, п. 36, теорема 1], заключаем, что $Y(x, \lambda)=0, x \in \Gamma$. Отсюда следует, что $f(x)=0$ п.в. на Г. Ортогональность собственных функций, соответствующих различным собственным значениям, вытекает из теоремы $3 ; \Upsilon-1$ и $\Upsilon$ собственных функций, соответствующих каждому из собственных значений множеств $\Omega_{\Upsilon}^{\mathrm{II}}$ и $\Omega_{\Upsilon}^{\mathrm{III}}$, ортогональны по построению (следствия б) и в) к теореме 4). Теорема доказана.

Пусть $\left\{\lambda_{n}\right\}_{n} \geqslant 1$ - множество всех собственных значений краевой задачи (1)-(3) с учетом кратности, упорядоченных по возрастанию. Пусть $\left\{y_{n}(x)\right\}_{n \geqslant 1}-$ множество собственных функций $y_{n}(x)$, соответствующих собственным значениям $\lambda_{n}$ (кратным собственным значениям соответствуют собственные функции, упомянутые в следствии из теоремы 4 и взятые в произвольном порядке).

Теорема 8. Для любой абсолютно непрерывной функиии $f(x), x \in \Gamma$, имеет место разложение в обобщенный ряд Фуръе по собственным функииям $\left\{y_{n}(x)\right\}_{n \geqslant 1}$ краевой задачи (1)-(3):

$$
f(x)=\sum_{n=1}^{\infty} a_{n} y_{n}(x), \quad a_{n}=\int_{\Gamma} f(t) y_{n}(t) d t,
$$

причем ряд сходится равномерно на Г.

ДокАЗАТЕЛЬСтво. Пусть $f(x)$ - произвольная абсолютно непрерывная на $\Gamma$ функция. Функция $Y(x, \lambda)=\int_{\Gamma} G\left(x, t, \lambda_{n}\right) f(t) d t$ примет вид:

$$
\begin{aligned}
Y(x, \lambda)_{\gamma_{k}}= & \frac{1}{\lambda \Delta_{k}(\lambda)}\left(\varphi_{m}(x, \lambda) \int_{0}^{x}\left[-\varphi_{k}^{\prime \prime}(t, \lambda)+q(t) \varphi_{k}(t, \lambda)\right] f(t) d t\right. \\
& +\varphi_{k}(x, \lambda) \int_{x}^{\pi / 2}\left[-\varphi_{m}^{\prime \prime}(t, \lambda)+q(t) \varphi_{m}(t, \lambda)\right] f(t) d t \\
& \left.-\frac{v(\pi / 2, \lambda) D_{k}(\lambda)}{D(\lambda)} \varphi_{k}(x, \lambda) \int_{\gamma_{k}}\left[-\varphi_{k}^{\prime \prime}(t, \lambda)+q(t) \varphi_{k}(t, \lambda)\right] f(t) d t\right) \\
& -\frac{\varphi_{k}(x, \lambda)}{\lambda D(\lambda)} \theta_{k}(x, \lambda), \quad k=\overline{1, m-1}, \\
Y(x, \lambda)_{\gamma_{m}}= & \frac{1}{\lambda \Delta_{m}(\lambda)}\left(\varphi_{m}(x, \lambda) \int_{\pi / 2}^{x}\left[-\varphi_{1}^{\prime \prime}(t, \lambda)+q(t) \varphi_{1}(t, \lambda)\right] f(t) d t\right. \\
& +\varphi_{1}(x, \lambda) \int_{x}^{\pi}\left[-\varphi_{m}^{\prime \prime}(t, \lambda)+q(t) \varphi_{m}(t, \lambda)\right] f(t) d t \\
& \left.-\frac{D_{m}(\lambda)}{D(\lambda)} \varphi_{m}(x, \lambda) \int_{\gamma_{m}}\left[-\varphi_{m}^{\prime \prime}(t, \lambda)+q(t) \varphi_{m}(t, \lambda)\right] f(t) d t\right) \\
& -\frac{\varphi_{m}(x, \lambda)}{\lambda D(\lambda)} \theta_{m}(x, \lambda),
\end{aligned}
$$


где

$$
\begin{aligned}
\theta_{k}(x, \lambda)= & v\left(\frac{\pi}{2}, \lambda\right) \sum_{\substack{j=1 \\
(j \neq k)}}^{m-1} \prod_{\substack{i=1 \\
i \neq j, k)}}^{m-1} u_{i}\left(\frac{\pi}{2}, \lambda\right) \int_{\gamma_{j}}\left[-\varphi_{j}^{\prime \prime}(t, \lambda)+q(t) \varphi_{j}(t, \lambda)\right] f(t) d t \\
& +\prod_{\substack{i=1 \\
(i \neq k)}}^{m-1} u_{i}\left(\frac{\pi}{2}, \lambda\right) \int_{\gamma_{m}}\left[-\varphi_{m}^{\prime \prime}(t, \lambda)+q(t) \varphi_{m}(t, \lambda)\right] f(t) d t, \\
\theta_{m}(x, \lambda)= & \sum_{j=1}^{m-1} \prod_{\substack{i=1 \\
(i \neq j)}}^{m-1} u_{i}\left(\frac{\pi}{2}, \lambda\right) \int_{\gamma_{j}}\left[-\varphi_{j}^{\prime \prime}(t, \lambda)+q(t) \varphi_{j}(t, \lambda)\right] f(t) d t .
\end{aligned}
$$

Учитывая вид (7) функций $\varphi_{k}(x, \lambda)$, в представлении функции $Y(x, \lambda), x \in \Gamma$, вычислим по частям интегралы, содержащие вторые производные от $\varphi_{k}(x, \lambda)$ :

$$
Y(x, \lambda)_{\Gamma}=\frac{f(x)}{\lambda}+\frac{1}{\lambda}\left(S_{1}(x, \lambda)+S_{2}(x, \lambda)\right),
$$

здесь

$$
\begin{aligned}
S_{1}(x, \lambda)_{\gamma_{k}}= & \frac{1}{\Delta_{k}(\lambda)}\left(\varphi_{m}(x, \lambda) \int_{0}^{x} \varphi_{k}^{\prime}(t, \lambda) f^{\prime}(t) d t+\varphi_{k}(x, \lambda) \int_{x}^{\pi / 2} \varphi_{m}^{\prime}(t, \lambda) f^{\prime}(t) d t\right. \\
& \left.\quad-\frac{v(\pi / 2, \lambda) D_{k}(\lambda)}{D(\lambda)} \varphi_{k}(x, \lambda) \int_{\gamma_{k}} \varphi_{k}^{\prime}(t, \lambda) f^{\prime}(t) d t\right) \\
& \quad-\frac{\varphi_{k}(x, \lambda)}{D(\lambda)} \widehat{\theta}_{k}(x, \lambda), \quad k=\overline{1, m-1}, \\
S_{1}(x, \lambda)_{\gamma_{m}}= & \frac{1}{\Delta_{m}(\lambda)}\left(\varphi_{m}(x, \lambda) \int_{\pi / 2}^{x} \varphi_{1}^{\prime}(t, \lambda) f^{\prime}(t) d t+\varphi_{1}(x, \lambda) \int_{x}^{\pi} \varphi_{m}^{\prime}(t, \lambda) f^{\prime}(t) d t\right. \\
& \left.\quad-\frac{D_{m}(\lambda)}{D(\lambda)} \varphi_{m}(x, \lambda) \int_{\gamma_{m}} \varphi_{1}^{\prime}(t, \lambda) f^{\prime}(t) d t\right)-\frac{\varphi_{m}(x, \lambda)}{D(\lambda)} \widehat{\theta}_{m}(x, \lambda),
\end{aligned}
$$

где

$$
\begin{aligned}
\widehat{\theta}_{k}(x, \lambda)= & v\left(\frac{\pi}{2}, \lambda\right) \sum_{\substack{j=1 \\
(j \neq k)}}^{m-1} \prod_{\substack{i=1 \\
(i \neq j, k)}}^{m-1} u_{i}\left(\frac{\pi}{2}, \lambda\right) \int_{\gamma_{j}} \varphi_{j}^{\prime}(t, \lambda) f^{\prime}(t) d t \\
& +\prod_{\substack{i=1 \\
i \neq k)}}^{m-1} u_{i}\left(\frac{\pi}{2}, \lambda\right) \int_{\gamma_{m}} \varphi_{m}^{\prime}(t, \lambda) f^{\prime}(t) d t, \\
\widehat{\theta}_{m}(x, \lambda)= & \sum_{j=1}^{m-1} \prod_{\substack{i=1 \\
(i \neq j)}}^{m-1} u_{i}\left(\frac{\pi}{2}, \lambda\right) \int_{\gamma_{j}} \varphi_{j}^{\prime}(t, \lambda) f^{\prime}(t) d t,
\end{aligned}
$$

функция $S_{2}(x, \lambda)_{\Gamma}$ представлена оставшимися слагаемыми $Y(x, \lambda)_{\Gamma}$, не содержащими производные $\varphi_{k}^{\prime}(t, \lambda), k=\overline{1, m}$. Из асимптотических формул (23) и вида функции $S_{2}(x, \lambda)_{\Gamma}$ можно получить следующую оценку при фиксированном $\delta>0$ и достаточно большом $\rho^{*}>0$ :

$$
\max _{x \in \Gamma}\left|S_{2}(x, \lambda)\right| \leqslant \frac{C}{|\rho|}, \quad \rho \in G_{\delta}, \quad \rho \mid \geqslant \rho^{*} .
$$


Рассмотрим функцию $S_{1}(x, \lambda)_{\Gamma}$. Предположим сначала, что $f(x)$ абсолютно непрерывна на каждом из ребер $\gamma_{k}, k=\overline{1, m}$. Обозначим $g(x)=f^{\prime}(x)$, $x \in \Gamma$ (производные в точке $\pi / 2$ односторонние). Тогда интегрирование по частям приводит $S_{1}(x, \lambda)$ к виду, содержащему под знаком производной только функцию $g(x)$. В силу асимптотических формул (23) получаем оценку, аналогичную (31):

$$
\max _{x \in \Gamma}\left|S_{1}(x, \lambda)\right| \leqslant \frac{C}{|\rho|}, \quad \rho \in G_{\delta}, \quad|\rho| \geqslant \rho^{*} .
$$

Пусть теперь $g(x) \in L(\Gamma)$. Зафиксируем $\varepsilon>0$ и выберем абсолютно непрерывную функцию $g_{\varepsilon}(x)$ так, чтобы

$$
\int_{\Gamma}\left|g(t)-g_{\varepsilon}(t)\right| d t<\frac{\varepsilon}{2 \widetilde{C}}, \quad \widetilde{C}=\max _{x \in \Gamma} \sup _{\rho \in G_{\delta}}\left|S_{1}(x, \lambda)\right| .
$$

Тогда при $\rho \in G_{\delta}, \rho \geqslant \rho^{*}$ имеем

$$
\max _{x \in \Gamma}\left|S_{1}(x, \lambda)\right| \leqslant \max _{x \in \Gamma}\left|S_{1}\left(x, \lambda, q_{\varepsilon}\right)\right|+\max _{\Gamma}\left|S_{1}\left(x, \lambda, g-g_{\varepsilon}\right)\right| \leqslant \frac{\varepsilon}{2}+\frac{\widetilde{C}}{|\rho|} .
$$

Следовательно, существует $\rho^{0}>0$ такое, что $\max _{\Gamma}\left|S_{1}(x, \lambda)\right| \leqslant \varepsilon$ при $\rho>\rho^{0}$. В силу произвольности $\varepsilon>0$ получаем

$$
\lim _{|\rho| \rightarrow \infty} \max _{x \in \Gamma}\left|S_{1}(x, \lambda)\right|=0, \quad \rho \in G_{\delta} .
$$

Рассмотрим контурный интеграл

$$
I_{N}(x)=\frac{1}{2 \pi i} \int_{R_{N}} Y(x, \lambda) d \lambda, \quad R_{N}=\left\{\lambda:|\lambda|=\left(N+\frac{1}{2}\right)^{2}\right\}, \quad 1 \leqslant N \in \mathbb{N}
$$

(обход против часовой стрелки). Из (31), (32) вытекает, что

$$
I_{N}(x)=f(x)+\varepsilon_{N}(x), \quad \lim _{N \rightarrow \infty} \max _{x \in \Gamma}\left|\varepsilon_{N}(x)\right|=0 .
$$

С другой стороны, можно вычислить $I_{N}(x)$ с помощью теоремы о вычетах [7; гл. II, §7, п. 26, теорема 1]. Рассмотрим следующие случаи (следствие из теоремы 4).

1. Пусть $\lambda_{n}=\lambda_{n}^{\mathrm{I}} \in \Omega^{\mathrm{I}}$. Тогда

$$
y\left(x, \lambda_{n}^{\mathrm{I}}\right)=\frac{1}{\sqrt{\widehat{\omega}_{n}^{\mathrm{I}}}} \widehat{\varphi}_{1}\left(x, \lambda_{n}^{\mathrm{I}} \beta_{n}^{\mathrm{I}}\right), \quad x \in \Gamma .
$$

Вычеты функции $Y(x, \lambda)_{\Gamma}$ в точках $\lambda_{n}^{\mathrm{I}}$ определяются формулой $(25)$ :

$$
\operatorname{Res}_{\lambda=\lambda_{n}^{\mathrm{I}}} Y(x, \lambda)_{\Gamma}=a\left(\lambda_{n}^{\mathrm{I}}\right) y\left(x, \lambda_{n}^{\mathrm{I}}\right), \quad a\left(\lambda_{n}^{\mathrm{I}}\right)=\int_{\Gamma} y\left(t, \lambda_{n}^{\mathrm{I}}\right) f(t) d t .
$$

Аналогично для $\lambda_{n}=\lambda_{n}^{\mathrm{III}} \in \Omega_{1}^{\mathrm{III}}$ получаем, что

$$
y\left(x, \lambda_{n}^{\mathrm{III}}\right)=\frac{1}{\sqrt{\widehat{\omega}_{n}^{\mathrm{III}}}} \widehat{\varphi}_{1}\left(x, \lambda_{n}^{\mathrm{III}} \beta_{n}^{\mathrm{III}}\right), \quad x \in \Gamma .
$$

Вычеты функции $Y(x, \lambda)_{\Gamma}$ в точках $\lambda_{n}^{\mathrm{III}}$ определяются формулой $(26)$ :

$$
\operatorname{Res}_{\lambda=\lambda_{n}^{\mathrm{III}}} Y(x, \lambda)_{\Gamma}=a\left(\lambda_{n}^{\mathrm{II}}\right) y\left(x, \lambda_{n}^{\mathrm{III}}\right), \quad a\left(\lambda_{n}^{\mathrm{III}}\right)=\int_{\Gamma} y\left(t, \lambda_{n}^{\mathrm{III}}\right) f(t) d t .
$$


2. Пусть $\lambda_{n}=\lambda_{n}^{\mathrm{III}} \in \Omega_{\Upsilon}^{\mathrm{III}}, \Upsilon=\overline{2, m-1}$. Каждому собственному значению $\lambda_{n}^{\mathrm{III}}$ соответствуют $\Upsilon$ собственных функций

$$
y_{k}\left(x, \lambda_{n}^{\mathrm{III}}\right)=\frac{1}{\sqrt{\int_{\Gamma} \chi_{k}^{2}\left(t, \lambda_{n}^{\mathrm{III}}\right) d t}} \chi_{k}\left(x, \lambda_{n}^{\mathrm{III}}\right), \quad k=\overline{1, \Upsilon}
$$

(следствие б) из теоремы 4); вычеты функции $Y(x, \lambda)_{\Gamma}$ в точках $\lambda_{n}^{\mathrm{III}}$ определяются формулой (27):

$$
\operatorname{Res}_{\lambda=\lambda_{n}^{\mathrm{III}}} Y(x, \lambda)_{\Gamma}=\sum_{k=1}^{\Upsilon} a_{k}\left(\lambda_{n}^{\mathrm{III}}\right) y_{k}\left(x, \lambda_{n}^{\mathrm{III}}\right), \quad a_{k}\left(\lambda_{n}^{\mathrm{III}}\right)=\int_{\Gamma} y_{k}\left(t, \lambda_{n}^{\mathrm{III}}\right) f(t) d t .
$$

3. Пусть $\lambda_{n}=\lambda_{n}^{\mathrm{II}} \in \Omega_{\Upsilon}^{\mathrm{II}}, \Upsilon=\overline{2, m-1}$. Каждому собственному значению $\lambda_{n}^{\mathrm{II}}$ соответствуют $\Upsilon-1$ собственных функций

$$
y_{k}\left(x, \lambda_{n}^{\mathrm{II}}\right)=\frac{1}{\sqrt{\int_{\Gamma} \chi_{k}^{2}\left(t, \lambda_{n}^{\mathrm{II}}\right) d t}} \chi_{k}\left(x, \lambda_{n}^{\mathrm{II}}\right), \quad k=\overline{1, \Upsilon-1}
$$

(следствие в) из теоремы 4); вычеты функции $Y(x, \lambda)_{\Gamma}$ в точках $\lambda_{n}^{\mathrm{II}}$ определяются формулой (28):

$$
\operatorname{Res}_{\lambda=\lambda_{n}^{\mathrm{II}}} Y(x, \lambda)_{\Gamma}=\sum_{k=1}^{\Upsilon-1} a_{k}\left(\lambda_{n}^{\mathrm{II}}\right) y_{k}\left(x, \lambda_{n}^{\mathrm{II}}\right), \quad a_{k}\left(\lambda_{n}^{\mathrm{II}}\right)=\int_{\Gamma} y_{k}\left(t, \lambda_{n}^{\mathrm{II}}\right) f(t) d t .
$$

Множество $R_{N}$ ( $N$ фиксировано) содержит конечное число собственных чисел вида $\lambda_{n}^{\mathrm{I}}, \lambda_{n}^{\mathrm{II}}, \lambda_{n}^{\mathrm{III}}$. Отсюда, учитывая формулы (33)-(36), получаем, что $I_{N}(x)$ будет содержать конечное число слагаемых вида

$$
\begin{array}{cl}
a\left(\lambda_{n}^{\mathrm{I}}\right) y\left(x, \lambda_{n}^{\mathrm{I}}\right), & a\left(\lambda_{n}^{\mathrm{III}}\right) y\left(x, \lambda_{n}^{\mathrm{III}}\right), \quad\left(\lambda_{n}^{\mathrm{III}} \in \Omega_{1}^{\mathrm{III}}\right), \\
\sum_{k=1} a_{k}\left(\lambda_{n}^{\mathrm{II}}\right) y_{k}\left(x, \lambda_{n}^{\mathrm{II}}\right), & \sum_{k=1}^{m_{0}} a_{k}\left(\lambda_{n}^{\mathrm{III}}\right) y_{k}\left(x, \lambda_{n}^{\mathrm{III}}\right) \quad\left(\lambda_{n}^{\mathrm{III}} \in \Omega_{\Upsilon}^{\mathrm{III}}\right) .
\end{array}
$$

Перенумеровка коэффициентов $a\left(\lambda_{n}^{\mathrm{I}}\right), a\left(\lambda_{n}^{\mathrm{II}}\right), a_{k}\left(\lambda_{n}^{\mathrm{III}}\right)$ и переобозначение собственных функций в соответствии с кратностями собственных значений приводит к коэффициентам $a_{n}$ и собственным функциям $y_{n}(x)$, а последующий переход к пределу при $N \rightarrow \infty$ дает (29). Остается отметить, что система собственных функций $\left\{y_{n}(x)\right\}_{n} \geqslant 1$ образует ортонормальный базис в $L_{2}(\Gamma)$ : для $f(x) \in L_{2}(\Gamma)$ ряд $(29)$ сходится в $L_{2}(\Gamma)$, причем имеет место равенство Парсеваля

$$
\int_{\Gamma} f^{2}(x) d x=\sum_{n=1}^{\infty} a_{n}^{2}
$$

Теорема доказана.

В заключение приведем пример моделирования колебательных процессов упругой мачты с поддерживающими упругими растяжками. Остановимся на модели так называемой одноуровневой системы "мачта-растяжки", имеющей один узел закрепления растяжек к телу мачты $[2$; соотношения (25), (26)]. При этом мачтовый континуум, находящийся выше узла закрепления растяжек, имеет массу несравнимо меньшую, чем масса остальной части мачты, что 
дает возможность пренебречь ею в модели. Если же учитывать указанное обстоятельство, то мачтовый континуум, находящийся выше узла закрепления растяжек, интерпретируется как точка узла закрепления растяжек, несущая сосредоточенную массу, равную массе этого континуума, и для исследования необходимо привлекать теорию дифференциальных уравнений с производными по мере [3; гл. 7]. Континуум, находящийся ниже узла закрепления растяжек, испытывает продольные колебания.

Пусть многообразие $\Im$ - граф-звезда с ребрами $\Im_{k}, k=\overline{1, m}$, и узлом $\xi$, и пусть каждое ребро $\Im_{k}, k=\overline{1, m-1}$, параметризовано отрезком $[0, \ell / 2]$, а ребро $\Im_{m}$ - отрезком $[\ell / 2, \ell]$. Моделирование колебательного процесса осуществляется на графе $\Im$ и описывается для $(\zeta, t) \in \Im \times[0, T]$ соотношениями, состоящими из $m$ уравнений

$$
\frac{\partial^{2} \Omega}{\partial t^{2}}(\zeta, t)=\frac{\partial}{\partial \zeta}\left(a(\zeta) \frac{\partial \Omega}{\partial \zeta}(\zeta, t)\right)-b(\zeta) \Omega(\zeta, t)
$$

на каждом ребре $\Im_{k}, k=\overline{1, m}$, и уравнением в узле $\xi$ :

$$
a\left(\frac{\ell}{2}\right)_{\Im_{m}} \frac{\partial \Omega}{\partial \zeta}\left(\frac{\ell}{2}, t\right)_{\Im_{m}}-\sum_{k=1}^{m-1} a\left(\frac{\ell}{2}\right)_{\Im_{k}} \frac{\partial \Omega}{\partial \zeta}\left(\frac{\ell}{2}, t\right)_{\Im_{k}}=b\left(\frac{\ell}{2}\right)_{\Im_{m}} \Omega\left(\frac{\ell}{2}, t\right)_{\Im_{m}}
$$

(соотношения (37), (38) назовем уравнением колебания на графе э).

Для получения математической модели физического процесса естественным образом ставятся начальные условия при $\zeta \in \Im, t=0$ :

$$
\Omega(\zeta, 0)=\tau(\zeta), \quad \frac{\partial \Omega}{\partial t}(\zeta, 0)=\widehat{\tau}(\zeta)
$$

и граничные условия в точках $0 \in \Im_{k}, k=\overline{1, m-1}, \ell \in \Im_{m}$ (условия во внешних узлах графа $)$ :

$$
a(0)_{\Im_{k}} \frac{\partial \Omega}{\partial \zeta}(0, t)_{\Im_{k}}-h_{k} \Omega(0, t)_{\Im_{k}}=0, \quad a(\ell) \frac{\partial \Omega}{\partial \zeta}(\ell, t)+H \Omega(\ell, t)=0 .
$$

Применение метода Фурье к задаче (37)-(40) и последующие преобразования, аналогичные преобразованиям Лиувилля (см., например, [8; гл. II, § 1.2]) приводят к задаче Штурма-Лиувилля (1)-(3) на графе $\Gamma$, где потенциал $q(x)$ определяется через функции $a(\zeta), b(\zeta)$ : компоненты $q(x)_{\gamma_{k}}, k=\overline{1, m-1}$, определяют упругие характеристики поддерживающих растяжек, $q(x)_{\gamma_{m}}$ - упругие характеристики продольных колебаний мачты.

Обоснованием метода Фурье при отыскании решения граничной задачи (37)-(40) являются теоремы 7 и 8.

\section{Список литературы}

[1] М. Г. Завгородний, "Спектральная полнота корневых функций краевой задачи на графе", Докл. РАН, 335:3 (1994), 281-283; англ. пер.: M. G. Zavgorodniı̌, "Spectral completeness of the root functions of a boundary value problem on a graph", Russian Acad. Sci. Dokl. Math., 49:2 (1994), 298-302.

[2] В.В. Провоторов, "Математическое моделирование колебательных процессов поддерживающих растяжек упругой мачты", Вестн. Ворон. ун-та. Cep. Cucm. анализ и информ. техн., 2006, № 2, 28-35. 
[3] Ю. В. Покорный, О.М.Пенкин, В. Л. Прядиев, А. В. Боровских, К. П. Лазарев, С.А. Шабров, Дифференциальные уравнения на геометрических графах, Физматлит, М., 2004.

[4] Э. Ч. Титчмарш, Разложение по собственным функциям, связанные с дифберенииальными уравнениями второго порядка, ч. 1, ИЛ, М., 1960; пер. с англ.: E. C. Titchmarsh, Eigenfunction expansions associated with second-order differential equations, Clarendon Press, Oxford, 1946.

[5] Л.Я. Цлаф, Вариационное исчисление и интегральные уравнения, Наука, М., 1970.

[6] В. А. Юрко, Введение в теорию обратных спектральных задач, Физматлит, М., 2007.

[7] Б. В. Шабат, Введение в комплексный анализ. Часть I. Функиии одного переменного, Наука, М., 1976; фр. пер.: B. Chabat, Introduction à l'analyse complexe. Tome 1. Fonctions d'une variable, Traduit Russe Math., Mir, Moscow, 1990.

[8] А. Г. Костюченко, И. С. Саргсян, Распределение собственных значений: Самосопряженные обыкновенные дифференциальные операторы, Наука, М., 1979.

В. В. Провоторов (V. V. Provotorov)

Воронежский государственный университет

E-mail: zao_vpo@mail.ru
Поступила в редакцию 17.10.2007 и 19.05.2008 